\title{
THE TEMPORAL ORGANIZATION OF BEHAVIOR ON PERIODIC FOOD SCHEDULES
}

\author{
Alliston K. Reid, Gustavo Bacha, and Concepción Morán \\ EASTERN OREGON STATE COLLEGE AND \\ UNIVERSIDAD NACIONAL AUTÓNOMA DE MÉXICO
}

\begin{abstract}
Various theories of temporal control and schedule induction imply that periodic schedules temporally modulate an organism's motivational states within interreinforcement intervals. This speculation has been fueled by frequently observed multimodal activity distributions created by averaging across interreinforcement intervals. We tested this hypothesis by manipulating the cost associated with schedule-induced activities and the availability of other activities to determine the degree to which (a) the temporal distributions of activities within the interreinforcement interval are fixed or can be temporally displaced, (b) rats can reallocate activities across different interreinforcement intervals, and (c) noninduced activities can substitute for schedule-induced activities. Obtained multimodal activity distributions created by averaging across interreinforcement intervals were not representative of the transitions occurring within individual intervals, so the averaged multimodal distributions should not be assumed to represent changes in the subject's motivational states within the interval. Rather, the multimodal distributions often result from averaging across interreinforcement intervals in which only a single activity occurs. A direct influence of the periodic schedule on the motivational states implies that drinking and running should occur at different periods within the interval, but in three experiments the starting times of drinking and running within interreinforcement intervals were equal. Thus, the sequential pattern of drinking and running on periodic schedules does not result from temporal modulation of motivational states within interreinforcement intervals.

Key words: schedule-induced behavior, adjunctive behavior, polydipsia, temporal organization, temporal control, drinking, running, motivation, interim behavior, fixed-time schedule, rats
\end{abstract}

If a rat is placed in a rich environment on a periodic food schedule, such as a fixed-time (FT) 60-s schedule, its behavior becomes stereotyped and temporally entrained within the interreinforcement interval (IRI). For example, if a drinking tube and a running wheel are available, the commonly observed pattern within the IRI is to eat the food, then drink, run, and return to the feeder in anticipation of the next food presentation. When averaged across all IRIs, the temporal frequency distribution of each activity typically has a single peak (or mode), and the different activities (such as drinking and running) usually peak at different periods within the IRI. We will

Portions of this work were presented at the Winter Conference on Animal Learning and at the Annual Meeting of the Psychonomic Society. Special thanks to Bill Timberlake, Allen Neuringer, Ben Williams, and John Staddon for enlightening discussions of these data and to Chip Ettinger, Peter Killeen, and Bill Timberlake for comments on an earlier version of this paper. Address correspondence to Alliston K. Reid, Department of Psychology, Eastern Oregon State College, La Grande, Oregon 97850. call the averaged distributions multimodal when the various activities peak at different periods in the IRI, and unimodal distributions will refer to averaged distributions in which the various activities peak at approximately the same period in the IRI.

Similar multimodal activity distributions have been observed in pigeons and many other experimental subjects (see recent review in Reid \& Staddon, 1990). These activity distributions have received attention mostly from researchers interested in schedule-induced behavior, because the first activity after food consumption is typically schedule induced and occurs at elevated levels. Models of this stereotyped temporal pattern of activities have usually been models of schedule induction.

For example, one of the most comprehensive models is that of Staddon (1977), who argued that periodic reward schedules modulate the animal's motivational states across the interreinforcement interval, producing interim, facultative, and terminal motivational states. The interim state is represented by the first activity in the IRI after consuming the food and is said to be induced to elevated levels by the schedule. 
The facultative state is represented by subsequent activities, such as running, that do not occur at elevated levels and are not considered to be schedule-induced activities. The terminal state refers to those activities related to obtaining reinforcement, such as operant behavior and searching around the feeder. Terminal activities are called "focal search" by Timberlake and his colleagues (Lucas, Timberlake, \& Gawley, 1988) to indicate that their function is normally associated with searching for reinforcement, and is the dominant, highest priority activity in periodic schedules (Reid \& Dale, 1983; Reid, Piñones Vazquez, \& Alatorre Rico, 1985). Other models of schedule induction similarly have accounted for the temporal regularity of these activity distributions by assuming that periodic reward schedules temporally modulate the motivational states of the animal within the IRI (Alferink, Bartness, \& Harder, 1980; Cohen, Looney, Campagnoni, \& Lawler, 1985; Falk, 1971; Killeen, 1975, 1979; Osborne, 1978; Staddon \& Ayres, 1975; Thompson \& Lubinski, 1986; Wetherington \& Riley, 1986).

Many studies have demonstrated multimodal activity patterns in rats on a wide range of interval durations (see review in Reid \& Staddon, 1990). The multimodal pattern most often reported is: consume the food, then drink, run, and return to the feeder area in anticipation of the next food delivery. For example, Staddon and Ayres (1975) carried out a detailed analysis of the distributions within the IRI, and their Figure 2 (Rat 4) has often been republished as the classic example of the drinkrun pattern. Nevertheless, a careful reading of their paper indicates that time allocation within the IRI is not as stereotyped as this single figure might imply. Of the 5 rats in their study, Rat 4 was the most clear-cut example of the drink-run pattern. In all other cases (4 of the 5 subjects), the temporal distributions of running and drinking overlapped substantially. Because the rats could not run and drink at the same time within any individual IRI, this overlap indicates that the averaged temporal distributions may not accurately represent transitions between activities within any particular IRI. The temporal distributions of both activities started at approximately the same time within the interval, although they differed somewhat in form. Often either drinking or running, but not both, occurred in a given interval. Even so, the averaged distributions across the session were multimodal, with drinking preceding running, but there was substantial (often complete) overlap between the distributions.

Because many reinforcement schedules are time based or have prominent temporal components, it is of fundamental importance to understand the roles of these temporal components in producing or modulating the subject's motivational states. An understanding of the dynamics of any activity, including operant behavior, in schedules with prominent temporal components requires an understanding of the interaction of available activities and their associated motivational states (Hinson \& Staddon, 1978). Choice between activities and persistence would be difficult to predict if the subject's motivational states change in ways that are poorly understood. Clearly this concern is not restricted to studies involving schedule-induced behavior, because these stereotyped patterns of activities have been observed even in the absence of schedule-induced behavior (see Reid \& Staddon, 1990, for a recent review).

In three experiments, we used two approaches to determine whether periodic food schedules directly modulate the subject's motivational states within individual intervals to produce multimodal activity distributions. The first approach was to increase systematically the number of bar presses required for access to the first activity that normally followed consumption of the food pellet (usually the induced activity) until it was nearly eliminated. This manipulation allows other activities to gradually replace the costly activity. Examination of the resulting temporal distributions could help determine the roles that temporal cues play in behavior allocation and the extent to which schedule-induced activities are constrained to the period just after food delivery.

The second approach was to compare the temporal distributions of running and drinking within the IRI in situations in which the activities were not restricted versus various situations in which only a single activity was available for the entire IRI. This manipulation should help determine whether activities occur at periods within the interval because of a modulating influence of the periodic schedule or because of the previous expression of particular behavior. 


\section{EXPERIMENT 1}

What would constitute evidence that periodic food schedules modulate the subject's motivational states within each IRI? The stereotyped behavior patterns producing multimodal frequency distributions are usually considered to be the primary evidence of a sequence of motivational states. To what extent is this sequence of activities fixed and unchangeable? A direct influence of the schedule on the motivational states would imply that drinking and running should occur at different periods within the IRI and should be poor substitutes for each other. The preferred temporal position of one activity within the IRI should not be strongly affected by preventing the occurrence of the other activity. For example, if drinking is prevented from occurring early in the interval when it normally occurs, will rats substitute running for drinking? Does the periodic schedule increase only the motivation to drink after food delivery, so running should not take its place early in the interval? Does the periodic schedule influence the rat's motivational states in such a way that the normal drink-run pattern cannot be shifted to other patterns?

In Experiment 1, we systematically increased the cost associated with the first activity in the interval (the induced activity) until its level decreased nearly to zero. This manipulation provided the opportunity for other activities to replace the costly activity. Examination of the resulting temporal distributions could help to determine the roles that temporal cues play in behavior allocation and the extent to which schedule-induced activities (such as drinking) and non-induced activities (such as running) are substitutable. This manipulation should also provide evidence to test the hypothesis that periodic food schedules modulate the subject's motivational states within each IRI.

\section{METHOD}

\section{Subjects}

Five naive female hooded rats, reared in this lab from Charles River stock, were housed individually in one room with 24-hr light. Each rat was approximately 6 months old at the beginning of the study. Their weights were reduced to $80 \%$ of their free-feeding weights by restricting food intake over a period of 10

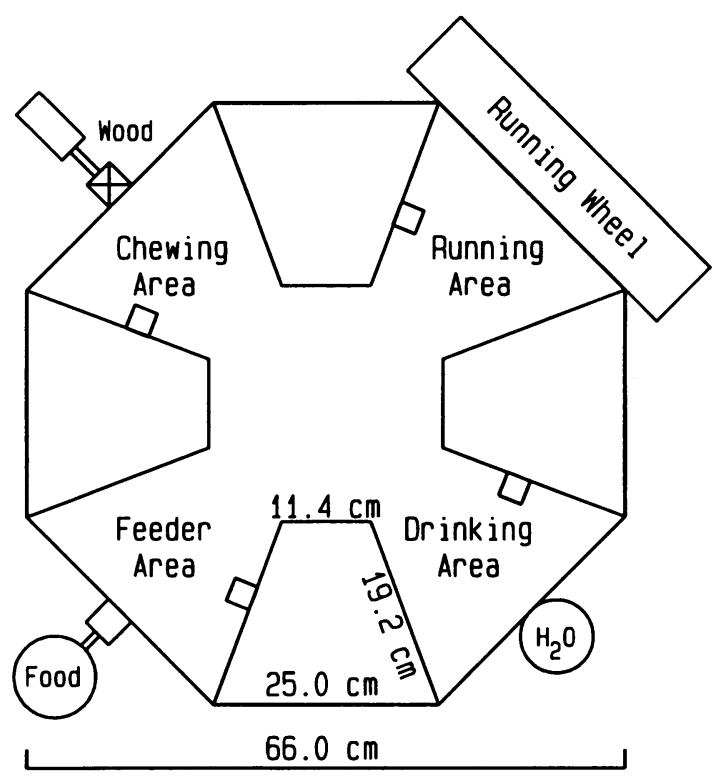

Fig. 1. Experimental apparatus used in Experiments 1,2 , and 3 .

days. Water was freely available in the home cages.

\section{Apparatus}

The octagonal apparatus (Figure 1) was used with four areas blocked off. Four areas were open: a feeder area, a drinking area containing a retractable drinking tube, a running area leading to a running wheel with a brake, and a chewing area containing a retractable $4.5 \mathrm{~cm}^{3}$ oak block. Each area contained a response lever located $6.4 \mathrm{~cm}$ above the hardware cloth floor. The tip of the metal drinking tube was recessed $0.5 \mathrm{~cm}$ behind the clear Plexiglas wall, and all except the tip was electrically insulated. The contact-lickometer circuitry required only $0.43 \mu \mathrm{A}$ for operation. The apparatus was located in a large homemade sound-attenuating chamber, and white noise at approximately $82 \mathrm{~dB}$ sound pressure level was present during all sessions. Noyes $45-\mathrm{mg}$ Formula $M$ pellets were dispensed throughout all experiments by a Gerbrands pellet dispenser.

An infrared light-emitting diode and phototransistor in the food hopper monitored headin and head-out of the hopper. Microswitches were connected to the rod holding the retractable chewing block in order to register movement of the block in any direction. Approxi- 
Table 1

Order of conditions and number of sessions in Experiment 1.

\begin{tabular}{|c|c|c|c|c|c|c|c|c|c|}
\hline \multicolumn{2}{|l|}{ Rat 1} & \multicolumn{2}{|l|}{ Rat 3} & \multicolumn{2}{|l|}{ Rat 7} & \multicolumn{2}{|l|}{ Rat 9} & \multicolumn{2}{|l|}{ Rat 10} \\
\hline 1. Massed food & 5 & Massed food & 5 & Massed food & 5 & Massed food & 5 & Massed food & 5 \\
\hline 2. FT $60 \mathrm{~s}$ & 11 & FT $60 \mathrm{~s}$ & 12 & FT $60 \mathrm{~s}$ & 12 & FT $60 \mathrm{~s}$ & 12 & FT $60 \mathrm{~s}$ & 12 \\
\hline 3. Free access & 35 & Free access & 34 & Free access & 36 & Free access & 36 & Free access & 36 \\
\hline 4. Shaping & 8 & Shaping & 8 & Shaping & 8 & Shaping & 8 & Shaping & 8 \\
\hline 5. Free access & 18 & Free access & 16 & Free access & 16 & Free access & 18 & Free access & 17 \\
\hline 6. FR 1 & 63 & FR 1 & 71 & FR 1 & 71 & FR 1 & 54 & FR 1 & 66 \\
\hline 7. FR 3 & 42 & FR 3 & 38 & FR 3 & 38 & Free access & 52 & FR 3 & 38 \\
\hline 8. FR 6 & 28 & FR 6 & 24 & FR 6 & 24 & FR 1 & 34 & FR 6 & 33 \\
\hline 9. FR 3 & 15 & FR 9 & 27 & FR 9 & 44 & FR 3 & 13 & FR 3 & 22 \\
\hline 10. FR 1 & 15 & FR 12 & 17 & FR 12 & 18 & FR 1 & 12 & FR 1 & 17 \\
\hline 11. FR 3 & 21 & FR 3 & 22 & FR 9 & 15 & FR 2 & 24 & FR 3 & 21 \\
\hline 12. FR 6 & 15 & FR 12 & 12 & FR 6 & 12 & FR 3 & 17 & FR 6 & 14 \\
\hline 13. FR 3 & 12 & FR 3 & 12 & FR 3 & 12 & FR 6 & 12 & FR 15 & 15 \\
\hline 14. FR 1 & 13 & FR 1 & 12 & FR 1 & 12 & FR 1 & 12 & FR 30 & 20 \\
\hline 15. & & & & & & & & FR 1 & 12 \\
\hline
\end{tabular}

Note. All conditions except the massed food condition contained an FT 60-s food schedule. The second condition above, labeled FT 60 s, allowed no access to wheel, water, or wood. Free access refers to FT $60 \mathrm{~s}$ with free access to wheel, water, and wood. In all FR conditions, access to the drinking tube was gained only by the completion of a fixed number of bar presses. Access to the running wheel and the chewing block required a single lever press on their respective levers in all FR conditions. After gaining access to any device, continued access was permitted until the interval was completed or until the subject earned access to a different device.

mately $0.2 \mathrm{~N}$ of force on the end of the wooden block in any direction was required to operate the microswitches. The chewing block could extend $4.5 \mathrm{~cm}$ into the chewing area, and when the chewing block was retracted, a metal door $\left(5.0 \mathrm{~cm}^{2}\right)$ automatically sealed off the block's entrance on the outside wall. The hardware cloth running wheel was $107.7 \mathrm{~cm}$ in circumference, and every half revolution operated a microswitch.

A microcomputer recorded every discrete event (from four levers, the drinking tube, the running wheel, the chewing block, head in hopper, and head out of hopper) and its time of occurrence with a resolution of $1 / 60 \mathrm{~s}$. These data were later transferred to another microcomputer for permanent storage and analysis.

\section{Procedure}

All subjects were exposed to six experimental phases after their body weights were reduced to $80 \%$ of their normal free-feeding weights. Table 1 summarizes each condition and the number of sessions in each for each subject. All sessions in all phases lasted $45 \mathrm{~min}$.

Phase 1: Massed food. Forty-five food pellets were placed in the feeder hopper at the beginning of each 45-min session. All events were recorded but had no programmed consequences. Each subject was exposed to this condition for five sessions.
Phase 2: FT 60 s no access. Food pellets were delivered individually on a fixed-time (FT) 60-s schedule independent of the subject's behavior. Subjects could enter all areas of the apparatus, but the drinking tube and chewing block were retracted and the running wheel brake was engaged during the entire session. Lever presses had no programmed consequences. All sessions began with a pellet delivery. This condition was in effect until the temporal distribution of head-in-feeder within the IRI appeared stable for each subject for at least eight sessions.

Phase 3: FT 60 s free access. Food pellets were delivered on an FT 60-s schedule as before, but subjects had free and continuous access to the drinking tube, running wheel, and the oak block. Lever presses were recorded but had no programmed consequences. All sessions began with a pellet delivery. The drinking tube, chewing block, and running wheel were presented to each subject at the beginning of the session with the first pellet delivery, and were retracted or locked at the end of the 45th interval. This condition was in effect until the session totals and the averaged temporal distributions of all activities within the IRI appeared stable for at least eight sessions for each subject.

Phase 4: Shaping. Each subject was deprived of water for $24 \mathrm{hr}$ and was then trained to press 
a lever for water delivery by a successive-approximations shaping procedure. During this procedure, each subject was confined to the drinking area of the apparatus. After each subject had responded on the water lever on a fixed-ratio (FR) 10 schedule for at least 50 water presentations, water was once again freely available in the home cages. Using the same shaping procedure and learning criterion, all subjects were then trained to press the lever in the wheel compartment for access to the running wheel, and the lever in the chewing area for access to the oak chewing block. The order of lever-press training to the various activities was the same for all subjects.

Phase 5: Free access recovery. Each subject was reexposed to the conditions of Phase 3 to ensure that lever-press training had not altered the temporal distributions of any activities within the IRI. The stability criteria of Phase 3 were used to terminate this condition.

Phase 6: FT 60 with FR for water. Each subject was exposed to the same FT 60-s schedule of food delivery, but an FR leverpress requirement was imposed for access to the schedule-induced activity. In Phase 3, drinking was the only schedule-induced activity for all animals, and it reliably occurred just after consumption of the food pellet. Therefore, access to the drinking tube was gained only by the completion of a fixed number of lever presses.

Every pellet delivery on the FT 60 -s schedule caused the drinking tube and the chewing block to be retracted and the running wheel to be locked. A single press of the corresponding lever in the wheel area or the chewing area resulted in immediate access to the corresponding device. Continued access was permitted until the 60-s interval was completed or until the subject pressed a lever that earned access to another activity. Lever presses in the feeder area were recorded but had no programmed consequences.

The FR value for access to the drinking tube was increased systematically across conditions until drinking was nearly eliminated. The ratio value was then decreased systematically to allow the opportunity for the previous drinking levels and temporal distributions to recover. This manipulation was repeated for some of the subjects. The actual FR values chosen varied considerably across subjects because they showed differential persistence in their drinking levels. Table 1 shows the order of the FR conditions (6 through 15) and the number of sessions in each for each subject. The stability criteria of Phase 3 were used to terminate each of the FR conditions.

\section{Results AND Discussion}

The temporal distributions of food-related behavior (head-in-feeder) quickly stabilized in Phase 2, when no access was allowed to the running wheel, oak chewing block, or the drinking tube. Roper, Edwards, and Crossland (1983) proposed that schedule-induced chewing may develop only if rats have ample exposure to the periodic food schedule before access to the oak block is allowed. The purpose of Phase 2 was simply to allow this exposure in order to encourage as many activities as possible to become induced.

The temporal distributions within IRIs stabilized more slowly in Phase 3 (FT $60 \mathrm{~s}$ with free access). The temporal distributions obtained in Phase 5 (free access recovery) accurately replicated the distributions obtained in Phase 3, so data from only Phase 5 will be shown. Schedule-induced chewing was not observed in any subject, and chewing levels were very low throughout the experiment. All subjects became polydipsic, with drinking exceeding the amounts observed during the massed-food condition of Phase 1. Temporal distributions, produced by taking second-bysecond averages across all IRIs, indicated that drinking occurred earliest in the interval, immediately after consumption of the pellet.

Data are reported for the last eight sessions for each subject in each experimental phase. Figure 2 shows the averaged session totals of licks and wheel turns as a function of the ratio value for access to the drinking tube in Phases 5 and 6. Circles identify the mean number of licks at the drinking tube per session as the ratio requirement was varied from no leverpress requirement (Phase 5: free access recovery), depicted as FR 0, to a maximum value of FR 30 for some subjects in Phase 6. The mean number of licks per session decreased systematically in all subjects as the ratio value increased.

Triangles in Figure 2 identify the mean number of wheel turns per session as a function of the ratio requirement for access to the drinking tube. For all subjects, wheel running decreased substantially with the imposition of the 


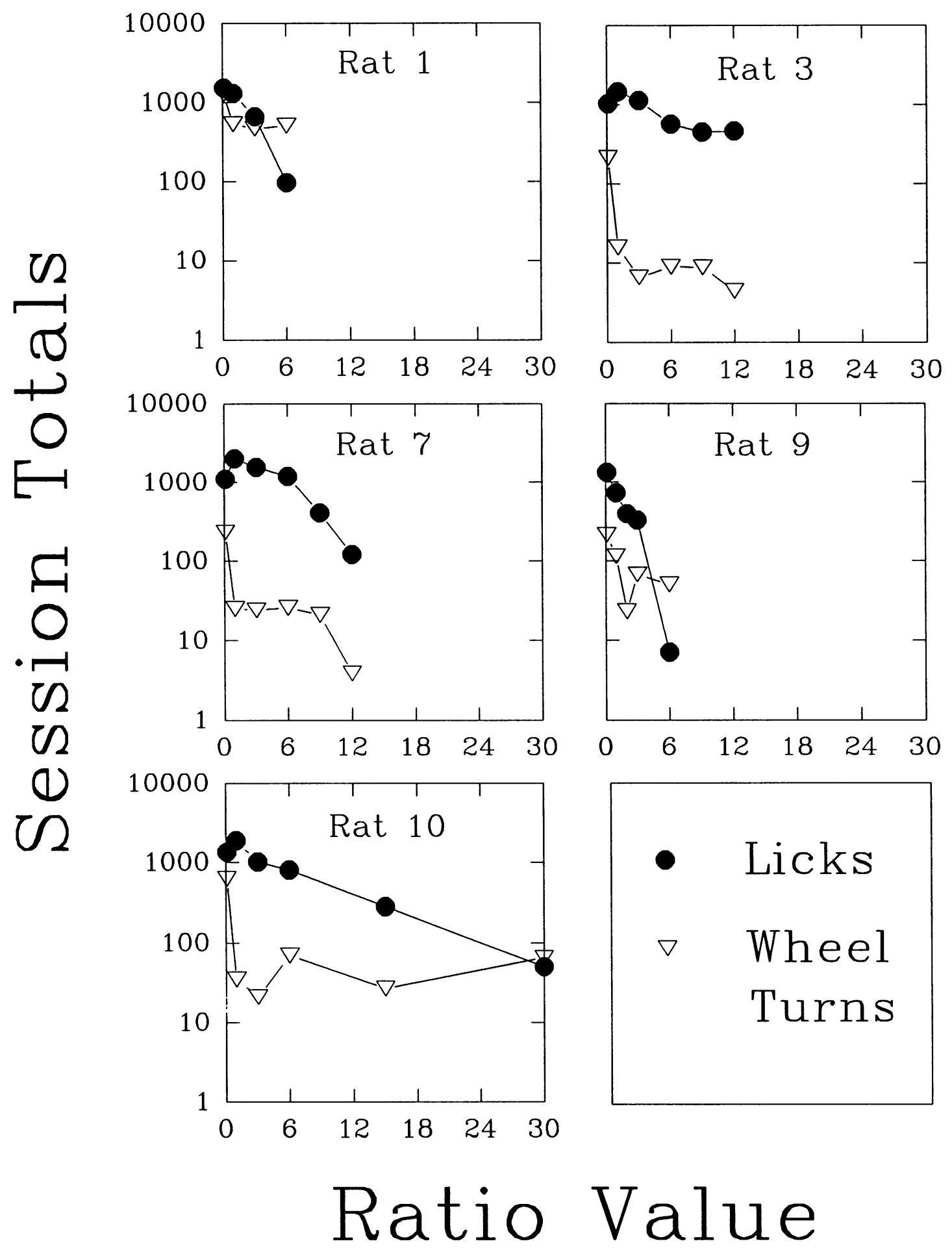

Fig. 2. Session totals of licks and wheel turns in Phases 5 and 6 of Experiment 1. The free access condition of Phase 5 is represented by a ratio value of 0 . The ratio value refers to the number of lever presses required to gain access to the drinking tube. Access to the running wheel required a single press of the wheel lever in each of the conditions of Phase 6. 


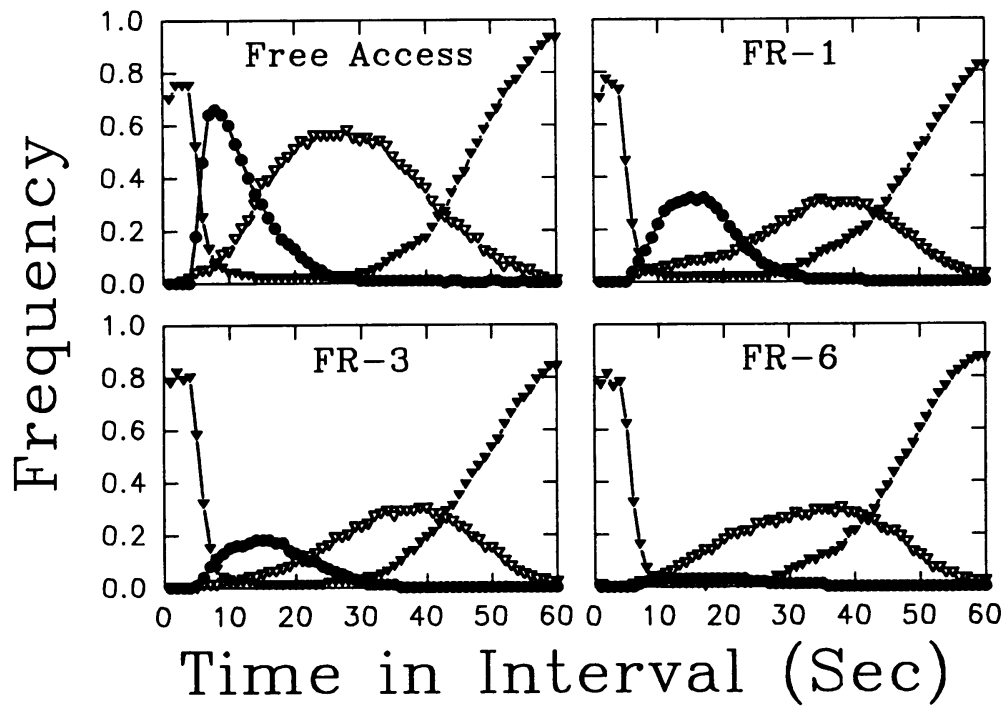

Fig. 3. Temporal frequency distributions of activities for Rat 1 occurring in Phase 5 (labeled free access) and the various fixed-ratio requirements of Phase 6. Filled circles represent drinking, filled triangles represent head-in-feeder, and open triangles represent wheel running. Each curve was generated by averaging across all IRIs in the last eight sessions of each condition.

single lever-press requirement to unlock the wheel. Running appeared to be unaffected by further increases in the ratio requirement for drinking. As drinking decreased across the FR conditions, running did not increase to take its place, even though more time within the interfood intervals was available for running when subjects finally stopped pressing the lever for water. Similarly, head-in-feeder (focal search) did not increase across the FR conditions, except for Rat 9.

As with the session totals, the temporal frequency distributions were also affected in the same way for all subjects as the FR value increased. For brevity, data from only a single subject are shown. Figure 3 depicts the temporal frequency distributions of activities across the 60-s IRI for each FR value for Rat 1. Each graph represents the total number of occurrences of each activity in 1-s bins during each IRI, summed across all IRIs in the last eight sessions of the condition and divided by the total number of IRIs in those sessions. The maximum possible on the ordinate is 1.0 , which would represent the occurrence of that activity during that second of every IRI in the last eight sessions. Chewing was rarely observed, so chewing distributions are not plotted.

The panel labeled "free access" in Figure 3 refers to the conditions of Phase 5 in which lever presses were not required for access to each activity on the FT 60 -s schedule. In all subjects, drinking reached its maximum level earlier in the interval than did running, with substantial overlap in their distributions.

As the FR value increased and drinking levels decreased, running did not shift to occupy the temporal periods in which drinking had occurred for any subject. Rather, the running distributions often shifted slightly to the right, toward the end of the interval. The running distributions did not shift left (earlier in the IRI) in any subject. No recorded activity replaced drinking in the early parts of the interval in 4 of the 5 animals. Rat 9 was the exception, whose level of head-in-feeder (focal search) increased as all other activities decreased.

The overlap in the drinking and running distributions in all subjects implies that these averaged frequency distributions do not accurately represent the temporal pattern of activities within some interfood intervals. Some IRIs could contain drinking without running, or vice versa, with bouts occurring at different parts of the interval. This finding implies that these averaged frequency distributions may not accurately reflect the changes in the subject's motivational states within individual IRIs. In order to examine this possibility, five interval 

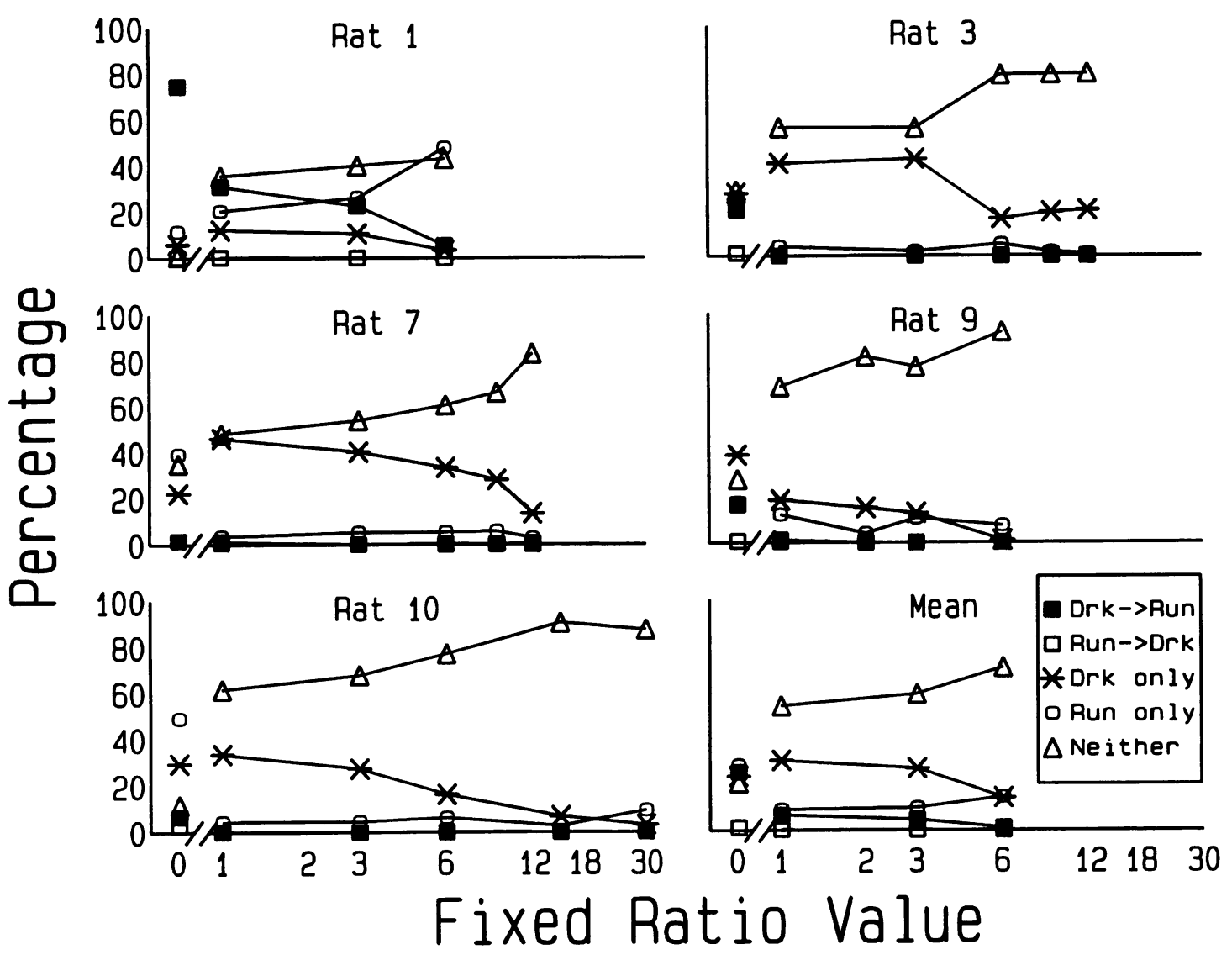

Fig. 4. Percentage of each interval type as a function of the lever-press requirement for access to the drinking tube in Phases 5 and 6. The free access condition of Phase 5 is represented by a fixed-ratio requirement of 0 . The interval types were distinguished by obtained transitions between activities.

types were defined: (a) IRIs in which the subjects drank and then ran, (b) IRIs in which the subjects ran then drank, (c) IRIs that contained drinking but no running, (d) IRIs that contained running but no drinking, and (e) IRIs that contained no drinking or running. Table 2 contains the percentages of each type of interval observed in each phase of Experiment 1.

The percentages of each type of IRI occurring in each condition, for each subject and the group mean, are presented in Figure 4 . The filled squares represent the interval type containing drinking preceding running. This percentage was relatively low in 4 of the 5 subjects in Phase 5 (when the levers were inoperative) and decreased further with increases in the ratio requirement for access to the drinking tube. Unfilled squares represent those intervals in which running preceded drinking and are below $3 \%$ in all cases. Unfilled circles represent intervals containing running with no drinking. These intervals were relatively constant with all ratio values and, in 4 of the 5 subjects (Rat 1 was the exception), were below the level observed in the free access condition of Phase 5. As the ratio value increased, the percentage of intervals containing neither running nor drinking (triangles) increased systematically, with the smallest increases shown by Rat 1 . The percentage of intervals containing drinking with no running (asterisks) decreased systematically in all rats.

The drink-run pattern commonly observed in averaged multimodal frequency distributions (cf. Reid \& Staddon, 1990; Staddon, 1977) clearly does not occur in every interval. These distributions are averaged across intervals that vary considerably in their composition and sequences of activities. In most subjects drinking 
Table 2

Percentage of each type of interval observed in each phase of Experiment 1.

\begin{tabular}{|c|c|c|c|c|c|c|}
\hline Condition & Interval type & Rat 1 & Rat 3 & Rat 7 & Rat 9 & Rat 10 \\
\hline $\begin{array}{l}\text { Free } \\
\text { access }\end{array}$ & $\begin{array}{l}\text { Drink } \rightarrow \text { run } \\
\text { Run } \rightarrow \text { drink } \\
\text { Drink only } \\
\text { Run only } \\
\text { No drink or run }\end{array}$ & $\begin{array}{r}74.7 \\
0.6 \\
11.7 \\
9.4 \\
3.6\end{array}$ & $\begin{array}{r}19.9 \\
1.1 \\
27.3 \\
23.3 \\
28.4\end{array}$ & $\begin{array}{r}1.7 \\
1.7 \\
22.2 \\
39.2 \\
35.2\end{array}$ & $\begin{array}{r}16.5 \\
0.6 \\
38.1 \\
17.3 \\
27.5\end{array}$ & $\begin{array}{r}6.8 \\
2.8 \\
29.5 \\
49.1 \\
11.8\end{array}$ \\
\hline FR 1 & $\begin{array}{l}\text { Drink } \rightarrow \text { run } \\
\text { Run } \rightarrow \text { drink } \\
\text { Drink only } \\
\text { Run only } \\
\text { No drink or run }\end{array}$ & $\begin{array}{r}31.1 \\
0.4 \\
12.3 \\
20.5 \\
35.7\end{array}$ & $\begin{array}{r}0.0 \\
0.0 \\
40.2 \\
4.0 \\
55.8\end{array}$ & $\begin{array}{r}1.3 \\
0.7 \\
46.2 \\
3.8 \\
48.2\end{array}$ & $\begin{array}{r}1.1 \\
0.3 \\
18.5 \\
2.2 \\
67.9\end{array}$ & $\begin{array}{r}0.2 \\
0.1 \\
33.6 \\
4.5 \\
61.6\end{array}$ \\
\hline FR 3 & $\begin{array}{l}\text { Drink } \rightarrow \text { run } \\
\text { Run } \rightarrow \text { drink } \\
\text { Drink only } \\
\text { Run only } \\
\text { No drink or run }\end{array}$ & $\begin{array}{r}22.7 \\
0.1 \\
10.7 \\
26.3 \\
40.2\end{array}$ & $\begin{array}{r}0.0 \\
0.1 \\
42.2 \\
2.0 \\
55.7\end{array}$ & $\begin{array}{r}0.1 \\
0.1 \\
40.3 \\
5.4 \\
54.1\end{array}$ & $\begin{array}{r}0.0 \\
0.0 \\
12.8 \\
0.7 \\
76.5\end{array}$ & $\begin{array}{r}0.1 \\
0.0 \\
27.4 \\
4.6 \\
67.8\end{array}$ \\
\hline FR 6 & $\begin{array}{l}\text { Drink } \rightarrow \text { run } \\
\text { Run } \rightarrow \text { drink } \\
\text { Drink only } \\
\text { Run only } \\
\text { No drink or run }\end{array}$ & $\begin{array}{r}5.5 \\
0.0 \\
3.4 \\
47.9 \\
43.2\end{array}$ & $\begin{array}{r}0.0 \\
0.0 \\
16.2 \\
5.1 \\
78.7\end{array}$ & $\begin{array}{r}0.0 \\
0.4 \\
33.4 \\
5.5 \\
60.7\end{array}$ & $\begin{array}{r}0.0 \\
0.0 \\
0.9 \\
7.4 \\
91.7\end{array}$ & $\begin{array}{r}0.3 \\
0.0 \\
16.3 \\
6.3 \\
77.1\end{array}$ \\
\hline FR 9 & $\begin{array}{l}\text { Drink } \rightarrow \text { run } \\
\text { Run } \rightarrow \text { drink } \\
\text { Drink only } \\
\text { Run only } \\
\text { No drink or run }\end{array}$ & & $\begin{array}{r}0.0 \\
0.3 \\
19.0 \\
1.7 \\
79.0\end{array}$ & $\begin{array}{r}0.0 \\
0.1 \\
28.0 \\
5.8 \\
66.1\end{array}$ & & \\
\hline FR 12 & $\begin{array}{l}\text { Drink } \rightarrow \text { run } \\
\text { Run } \rightarrow \text { drink } \\
\text { Drink only } \\
\text { Run only } \\
\text { No drink or run }\end{array}$ & & $\begin{array}{r}0.0 \\
0.0 \\
19.9 \\
0.9 \\
79.2\end{array}$ & $\begin{array}{r}0.0 \\
0.6 \\
13.4 \\
2.8 \\
83.2\end{array}$ & & \\
\hline FR 15 & $\begin{array}{l}\text { Drink } \rightarrow \text { run } \\
\text { Run } \rightarrow \text { drink } \\
\text { Drink only } \\
\text { Run only } \\
\text { No drink or run }\end{array}$ & & & & & $\begin{array}{r}0.0 \\
0.0 \\
6.8 \\
2.6 \\
90.6\end{array}$ \\
\hline FR 30 & $\begin{array}{l}\text { Drink } \rightarrow \text { run } \\
\text { Run } \rightarrow \text { drink } \\
\text { Drink only } \\
\text { Run only } \\
\text { No drink or run }\end{array}$ & & & & & $\begin{array}{r}0.0 \\
0.0 \\
3.1 \\
9.4 \\
87.5\end{array}$ \\
\hline
\end{tabular}

and running occupied the same interval in only a relatively small percentage of the total intervals $(M=23.4 \%$ in Phase $5, M=6.7 \%$ with FR 1 in Phase 6). In 4 of the 5 subjects, most intervals contained drinking without running, running without drinking, or neither activity. Rat 1 was an exception again, but only in the free access condition, with $74.7 \%$ of the intervals consisting of drinking followed by running. This percentage decreased substantially in later conditions. Rat 7 represented the opposite extreme, in which drinking preceded running in only $1.7 \%$ of the intervals.
When both drinking and running did occur in the same interval, running only rarely preceded drinking $(M=1.4 \%$ in Phase $5, M=$ $0.3 \%$ with FR 1 in Phase 6). Although the drink-run pattern was observed much more frequently than the run-drink pattern, many intervals were composed of drinking only $(M$ $=25.8 \%$ in Phase $5, M=30.2 \%$ with FR 1 in Phase 6$)$ or running only $(M=27.7 \%$ in Phase $5, M=9.0 \%$ with FR 1 in Phase 6). Intervals containing neither drinking nor running were also frequently observed $(M=22.6 \%$ in Phase $5, M=53.8 \%$ with FR 1 in Phase 


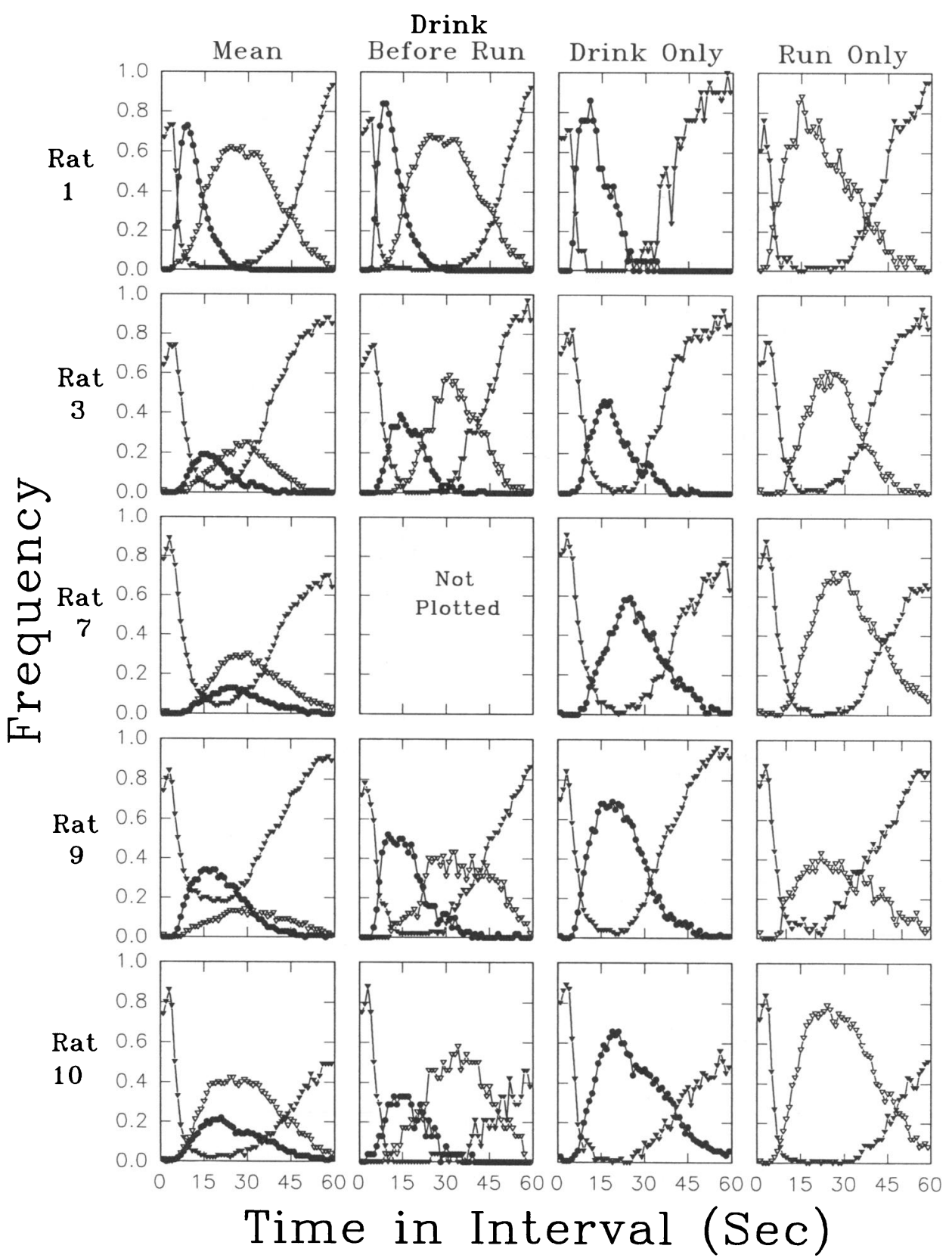

Fig. 5. Temporal frequency distributions of activities for all subjects in Phase 5. Filled circles represent drinking, filled triangles represent head-in-feeder, and open triangles represent wheel running. These curves were generated by classifying five types of intervals based on the obtained transitions between activities within the IRI and averaging across only those IRIs of the same type. The maximum value on the ordinate for any activity is 1.0 , which would represent the occurrence of that activity at that second in every IRI of that type. The column labeled "Mean" contains the distributions averaged across all five interval types for each subject. The interval type in which running preceded drinking is not depicted because it occurred very infrequently in this phase.

6, increasing to $70.3 \%$ with FR 6). The asymmetry in the frequency of the drink-run and the run-drink patterns is examined in more detail below.
Figure 5 depicts the temporal frequency distributions averaged across all IRIs in the last eight sessions (in the panel labeled "Mean") and the temporal frequency distributions of 


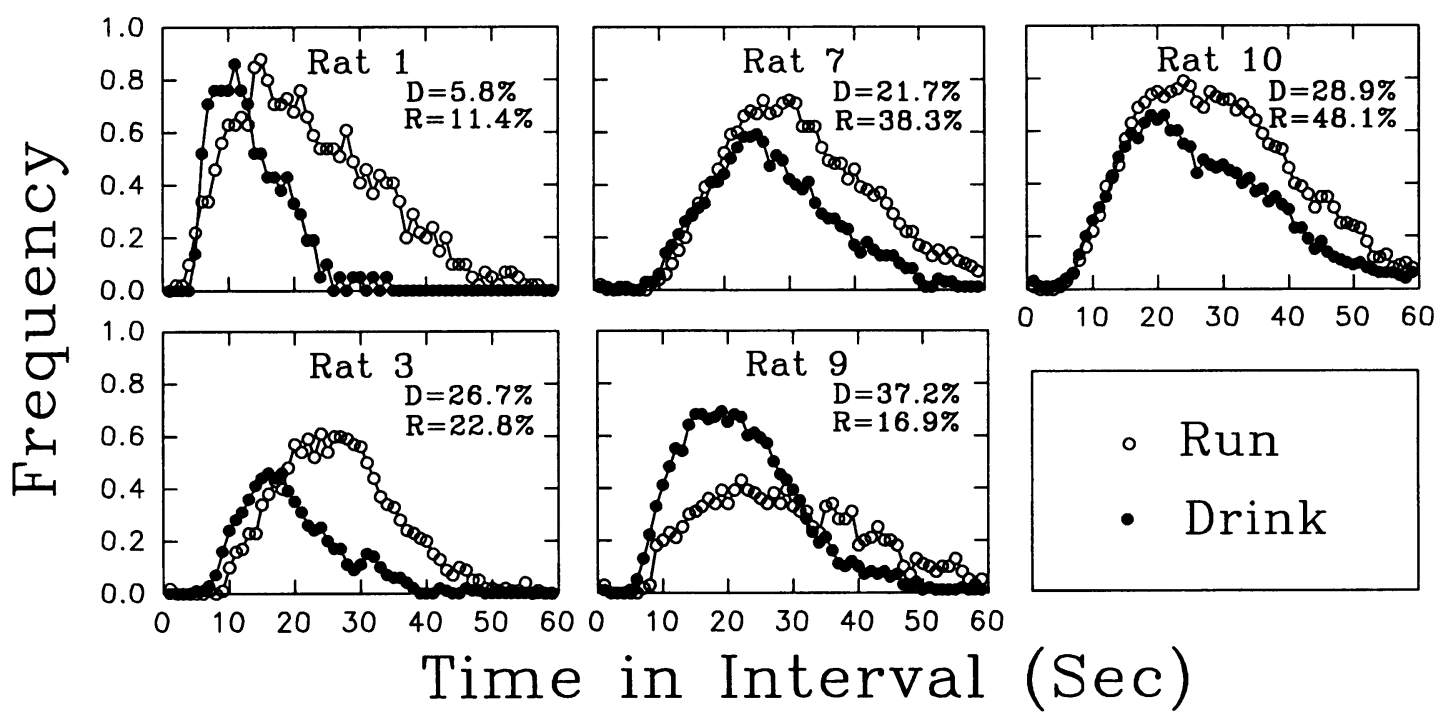

Fig. 6. Temporal frequency distributions of drinking and running that occurred in drinking-only and runningonly intervals for each subject in Phase 5 of Experiment 1. The drinking and running distributions are plotted together even though they occurred in separate IRIs in order to compare their temporal characteristics. The numbers in each panel represent the obtained percentages of drinking-only and running-only intervals.

each interval type for each rat in Phase 5. The mean distributions represent the total number of occurrences of each activity in 1-s bins during each IRI, summed across all IRIs in the last eight sessions of Phase 5 and divided by the total number of IRIs in those sessions (in order to normalize the ordinate). The maximum possible on the ordinate is 1.0 , which would represent the occurrence of that activity during that second of every IRI in the last eight sessions. In the remaining three panels, each distribution was generated by classifying five types of IRI based on the obtained transitions between activities within the IRI and taking second-by-second averages across only those IRIs of the same type. The distributions of those IRIs containing neither drinking nor running are not shown. Data were not plotted if the percentage of a particular interval type was below $2 \%$, because the resulting normalized distributions would appear highly irregular and would not be informative. The percentage of drink-before-run intervals was below $2 \%$ for Rat 7, so the data in that panel are not plotted. The interval type representing running before drinking was very low in all animals (see Table 2), so those panels are not plotted.

In all cases the mean distributions were composed of the individual IRIs that contrib- ute to the other panels. A second-by-second average of the individual IRIs used to create the distributions in each of the three panels representing particular interval types (and including the IRIs that contained neither drinking nor running and those infrequent run-thendrink IRIs) and divided by the total number of IRIs will exactly produce the averaged distributions in the panel labeled "Mean" (Figure 5).

In drink-then-run intervals, the total amount of time spent drinking and running was not greater than the time spent running in running-only intervals for any of the subjects. Thus the total amount of time engaged in activities unrelated to obtaining food was roughly constant across intervals, with running and drinking substituting for one another.

When drinking and running occurred in separate intervals, their respective temporal distributions were very similar in all subjects, as the two columns of panels on the right of Figure 5 show. The temporal distributions of drinking and running began at approximately the same time in the interval for each of the subjects.

The similarities between the drinking and running distributions for all subjects are illustrated in Figure 6. The data in Figure 6 are from intervals that contained drinking 


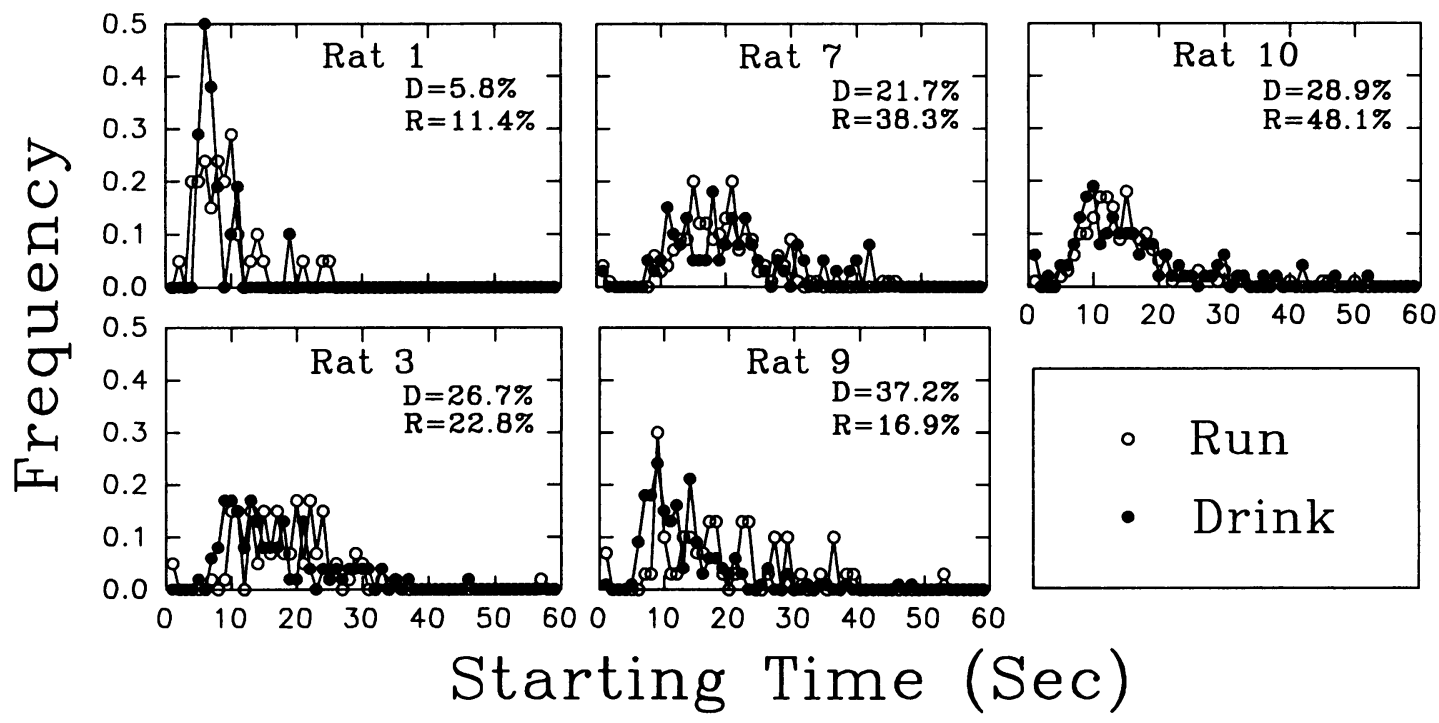

Fig. 7. Frequency distributions representing the times of onset of drinking and running obtained in drinking-only and running-only intervals for each subject in Phase 5 of Experiment 1 . The maximum value on the ordinate for either activity is 1.0 , which would represent the onset of that activity at that second in every IRI of that type.

without running or running without drinking, and are plotted together for comparison of their temporal characteristics. The numbers in each panel represent the percentages of intervals containing only drinking or only running. There was no consistent evidence that drinking and running tended to occur at different periods within the IRI.

Figure 6 also shows that drinking and running began at approximately the same time in the IRI for all rats in those intervals that contained only one of the activities. Drinking bouts were shorter in duration with less variation $(M=12.1 \mathrm{~s}, S D=6.3 \mathrm{~s})$ than running bouts $(M=19.5 \mathrm{~s}, S D=11.3 \mathrm{~s})$, so the distributions fell off with different slopes in the IRI. A more compelling demonstration of the striking similarities between their starting times can be made by examination of the frequency distributions of the onset times of running and drinking (Figure 7). From Figure 6 we saw that the starting time distributions should peak at approximately the same place, but it implied nothing about the actual shapes of the frequency distributions of onset times.

The frequency distributions of Figure 7 depict the variation in the time to the first response of drinking or running in those intervals in which only one of those activities occurred. The peaks of both distributions occur at approximately the same point within the IRI for all subjects. The overall forms of the two starting-time distributions are so similar in most subjects that they can be distinguished only with difficulty. It is not possible to demonstrate statistically that the distributions are the same, but the drinking and running distributions can be described as representing independent samples from the same hypothetical distribution with at least $95 \%$ certainty for all subjects, $t(58)=9.31,7.00,14.37,19.03,5.53$; $p<.05)$. The onset times of running and drinking were equivalent.

These results argue strongly against the notion that periodic food schedules directly modulate the tendencies to engage in drinking and running at different points in the IRI. Drinking and running usually occupied approximately the same portions of the IRI, and their onset times were nearly identical. Therefore, the multimodal activity distributions with rats obtained from averaging across all IRIs (cf. the panels labeled "Mean" in Figure 5 and the recent review in Reid \& Staddon, 1990) are results of averaging several types of intervals together: (a) intervals with drinking preceding running, (b) intervals with running preceding drinking, (c) intervals containing drinking but no running, (d) intervals containing running but no drinking, and (e) in- 
tervals containing neither drinking nor running.

The different shapes of the activity distributions are not produced by different onset times. We postulate that the rapid rise in the drinking distributions early in the interval results largely from licking reaching full speed quickly, whereas the inertia of the running wheel, frequent pauses within running bouts, and frequent reversals in the direction of running (from informal visual observations) often resulted in a more gradual increase in running speed and flatter distributions. For each subject, the drinking distributions declined earlier in the interval than did the running distributions because running bouts lasted longer than drinking bouts. Therefore drinking distributions peaked earlier than did the running distributions, even though the two activities began at the same point in the IRI. These distributions, and their resulting multimodal average, appear to be due to variables unique to each activity (e.g., inertia and preferred bout length) rather than a modulating influence on the rat's motivational states.

For two reasons, these data are not sufficient to rule out the possibility that periodic food schedules modulate the motivational states of the subject, producing the drink-run sequence seen in averaged data. First, this sequence was observed in a small number of intervals and was the dominant type in Rat 1 in one condition. For 4 of the 5 rats this drink-run pattern occurred in less than $20 \%$ of the intervals in Phase 5 but Rat 1 was an exception, drinking before running in nearly $75 \%$ of the intervals. Second, in those intervals that did contain both activities, the drink-run sequence was observed much more frequently than was the run-drink sequence. Running preceded drinking in less than $3 \%$ of all IRIs.

Various theories of schedule induction often imply that periodic reward schedules temporally modulate the subject's motivational states within every interfood interval, so transitions from drinking to running should occur in each IRI. This experiment demonstrated that the averaged multimodal activity distributions are not representative of the transitions that occur within most IRIs when each activity is continuously available. These averaged distributions should not be assumed to represent modulation of the motivational states of the subject within the IRI. Experiment 2 compares the temporal characteristics of similar frequency distributions in situations in which rats have continuous access to each activity with situations in which activities are not freely available.

\section{EXPERIMENT 2}

Experiment 1 attempted to determine the factors responsible for multimodal activity distributions by varying the cost associated with the first activity in the interfood interval. This manipulation offered the opportunity for other activities to replace the costly activity gradually. The question remains of how the time of onset of running and drinking depends upon the availability of the other activity. For example, Staddon (1977) argues that drinking and running compete with each other for available time in the interval, even though drinking is schedule induced and running is not. If drinking did not occur, would running take its place in the IRI? Does the periodic food schedule modulate the tendency to drink and run so that they will occupy different positions in the IRI, even if the other activity is not available?

In order to address these questions, Experiment 2 compared the observed, unrestricted temporal distributions with (a) the temporal distributions obtained when only a single subject-initiated activity was available for the entire interval and with (b) the temporal distributions obtained when the experimenter allowed access to only certain activities by locking the running wheel or retracting the drinking tube for the entire session. If periodic schedules modulate the motivational states of rats within the IRI, drinking and running should generally occupy different portions of the interval during both of these situations.

\section{METHOD}

\section{Subjects and Apparatus}

Five naive female hooded rats, reared in this lab from Charles River stock, were housed individually in one room with 24-hr light. Each rat was approximately 7 months old at the beginning of the study. Their ad lib weights were determined by averaging each subject's weight over 5 consecutive days. Their weights were reduced to $80 \%$ of their free-feeding weights by restricting food intake over a period of 10 days. Water was freely available in the home cages. 
Table 3

Order of conditions and number of sessions in Experiment 2.

\begin{tabular}{lrrrrr}
\hline \multicolumn{1}{c}{ Phase } & $\begin{array}{c}\text { Rat } \\
5\end{array}$ & $\begin{array}{c}\text { Rat } \\
6\end{array}$ & $\begin{array}{c}\text { Rat } \\
11\end{array}$ & $\begin{array}{c}\text { Rat } \\
12\end{array}$ & $\begin{array}{c}\text { Rat } \\
13\end{array}$ \\
\hline \hline 1. Massed food & 5 & 5 & 8 & 8 & 8 \\
2. FT 60 no access & 12 & 12 & 12 & 12 & 12 \\
3. FT 60 free access & 71 & 71 & 32 & 32 & 32 \\
4. Shaping & 8 & 8 & 8 & 8 & 8 \\
5. Phase 3 recovery & 12 & 12 & 12 & 12 & 12 \\
6. FR 1 each activity & 53 & 38 & 44 & 49 & 65 \\
7. Exclusive choice & 49 & 69 & 67 & 60 & 38 \\
8. Phase 6 recovery & 27 & 32 & 32 & 32 & 32 \\
9. FR 1 no choice: & & & & & \\
$\quad$ No water & 18 & - & 18 & - & - \\
$\quad$ No wheel & 18 & 18 & 17 & 17 & 16 \\
$\quad$ No water & - & 18 & - & 18 & died \\
10. Phase 3 recovery & 15 & 15 & 15 & 16 & - \\
\hline
\end{tabular}

The apparatus was the same as that used in Experiment 1.

\section{Procedure}

Table 3 summarizes the order of each condition and the number of sessions in each for each subject. Session duration was $45 \mathrm{~min}$ in all phases.

Phases 1 through 5. The conditions in each of these phases were the same as those of Phases 1 through 5 in Experiment 1.

Phase 6: FR 1 for each activity. Access to the drinking tube, running wheel, and the chewing block could be obtained only by a single lever press on their respective levers. A lever press on one lever resulted in retraction or locking of the other two devices. This FR 1 requirement was superimposed on the FT 60 -s food schedule. Transitions between activities within an interfood interval were allowed, but always required a lever press in the area with the new activity. Every pellet delivery retracted or locked each device until a subject earned access to it.

Phase 7: Exclusive choice. The lever-press requirement for each device was the same as in Phase 6. However, once a lever was pressed, all other levers became inoperative for the remaining portion of the interfood interval. Once a subject began one activity, transitions to the other two activities were not allowed for the rest of the interfood interval, forcing exclusive choice between activities. All devices were locked or retracted with every pellet delivery, and access to each could again be obtained by a single press on the appropriate lever.

Phase 8: FR 1 recovery. This phase replicated the conditions of Phase 6.

Phase 9: FR 1 no choice. The lever-press requirement for access to each activity was maintained, but either the running wheel was locked or the drinking tube was retracted for the entire session. The drinking tube was retracted first for 2 subjects, and the running wheel was locked first for the remaining 3 subjects. After this first condition, each subject was shifted to the other condition, in which the other apparatus was restricted and access was allowed to the previously restricted apparatus.

Phase 10: Free access recovery. This phase is a replication of Phases 3 and 5, in which levers had no scheduled consequences and access to each device was freely and continuously available.

\section{Results AND Discussion}

Data are reported for the last eight sessions for each subject in each experimental phase. Table 4 contains the total number of licks and wheel turns per session as a function of each experimental phase for each subject. All subjects showed a substantial increase in drinking level when shifted from the massed-food baseline (Phase 1) to the FT 60-s food schedule of Phase 3 . The drinking tube and running wheel had not been available in Phase 2. Running was not systematically affected by the shift in conditions: Running increased for 2 subjects, decreased for 2 subjects, and remained constant for 1 subject.

Phase 5 was a recovery of the conditions of Phase 3 and determined whether or not the intervening lever-press training procedure (Phase 4) altered the levels of drinking or wheel running. The levels of drinking and running in Phase 5 accurately recovered the levels observed in Phase 3 for all subjects.

The imposition of a single lever-press requirement for access to the drinking tube or the running wheel (Phase 6 ) resulted in a small reduction in running for all subjects. Drinking levels also diminished slightly for 4 of the 5 subjects, but the day-to-day variability in drinking levels exceeded this small difference.

When subjects were shifted to the exclusive choice condition (Phase 7) any IRI could contain drinking or running but not both. Subjects 
Table 4

Session totals of drinking and running in each condition of Experiment 2.

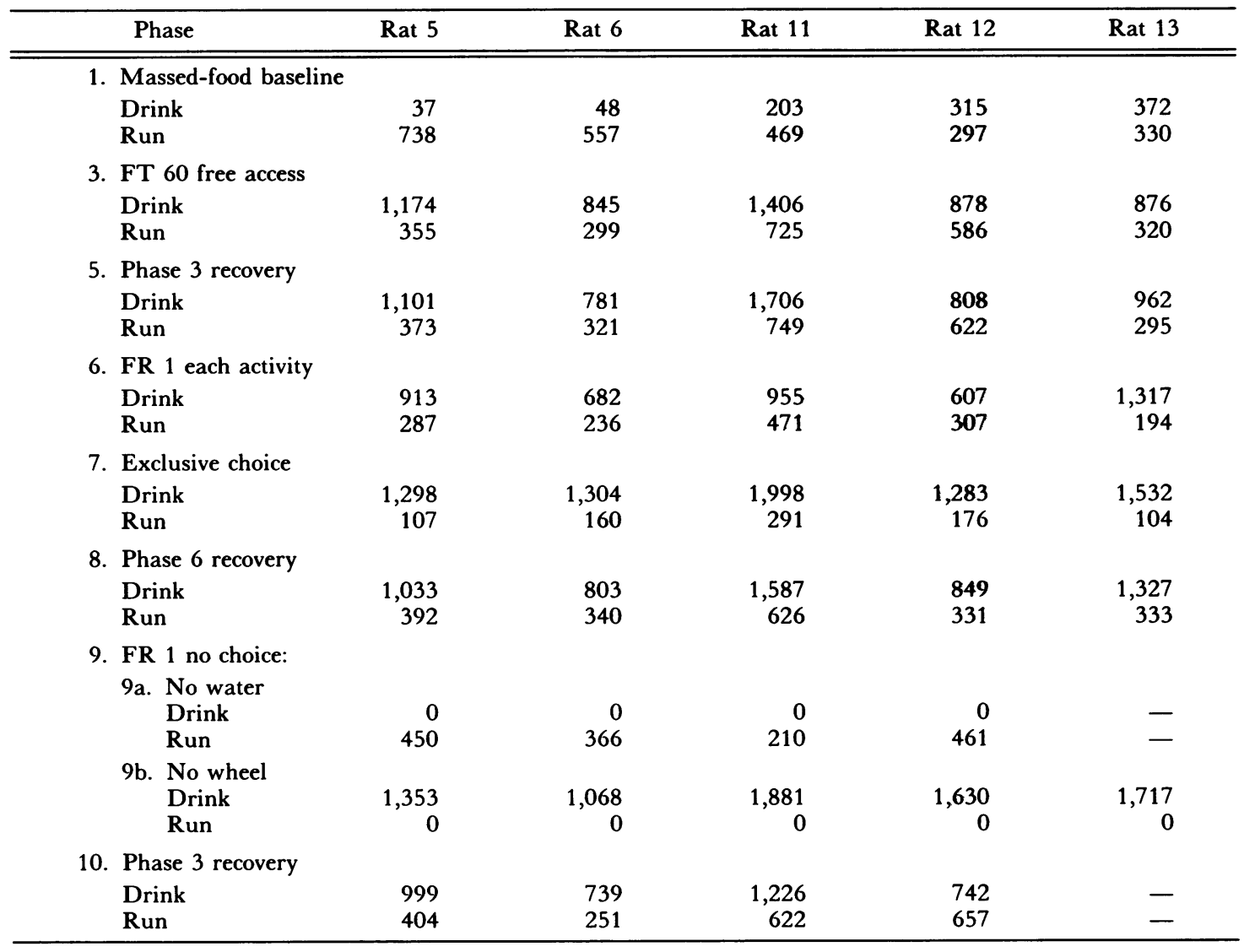

had to choose between drinking and running (or neither) in each interval. Drinking levels increased, often substantially, and running decreased in all subjects.

Phase 8 was a recovery of the conditions in Phase 6 in which a single lever-press requirement was in effect for access to the drinking tube or the running wheel. Drinking levels decreased and running increased to approximately recover the levels of Phase 6 in each subject.

In Condition A of Phase 9, the drinking tube was retracted for the entire session, and the running wheel became available after a single lever press. In Condition B, the running wheel brake was engaged, and the drinking tube was available after a single lever press. Running levels were not systematically affected by retracting the drinking tube. There was a small increase in running for 3 subjects and a sub- stantial decrease in another. (One subject, Rat 13, died before this phase was completed.) Drinking levels increased substantially in all subjects when the running wheel brake was engaged, compared to the previous condition in which both activities were available (Phase 8).

Phase 10 was a recovery of Phase 3 . Each of the 4 remaining subjects recovered the drinking and running levels observed in Phase 3.

Figure 8 depicts the frequency distributions of the starting times of drinking and running when they occurred alone in separate intervals in the conditions of Phase 5, which allowed free access to drinking and running on the FT 60-s food schedule. These conditions precisely replicated those of Phase 5 of Experiment 1, but with different subjects. As in Experiment 1 , statistical analysis revealed that the drinking and running distributions could be described 


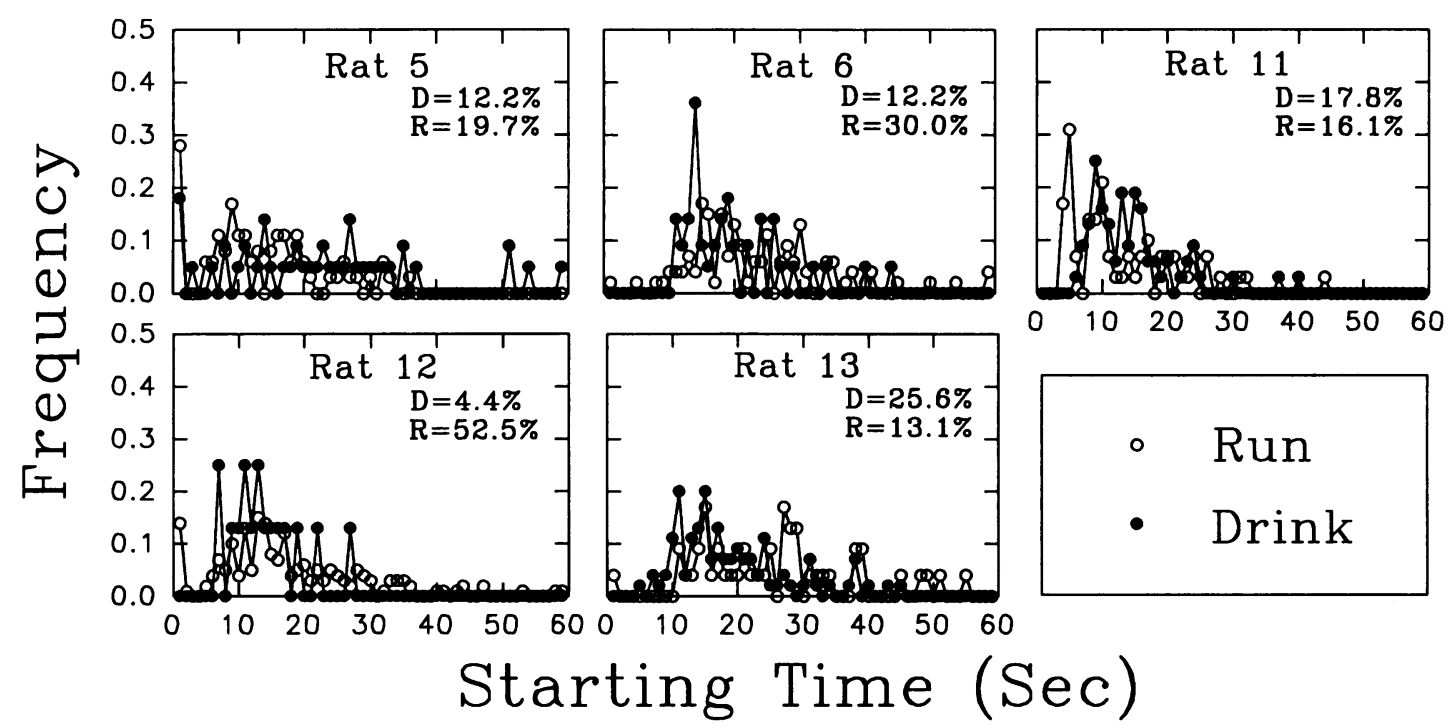

Fig. 8. Frequency distributions representing the times of onset of drinking and running obtained in drinking-only and running-only intervals in Phase 5 of Experiment 2. The maximum value on the ordinate for either activity is 1.0 , which would represent the onset of that activity at that second in every IRI of that type. The numbers in each panel represent the obtained percentages of drinking-only and running-only intervals.

as representing independent samples from the same hypothetical distribution with at least $95 \%$ certainty for all subjects, $t(58)=6.79$, $9.56,11.73,13.08,13.54 ; p<.05$. This similarity demonstrates that drinking and running began at the same point within the IRI in those intervals that contained one or the other, but not both. These results accurately replicate the findings of Experiment 1 (Figure 7).

In the exclusive choice condition (Phase 7), subjects could engage in drinking or running, but not both in any interval. Transitions between drinking and running were not possible within an interval. Figure 9 shows the corresponding temporal distributions of running and drinking within the separate intervals, plotted together for ease of comparison. Once again, the starting times of both activities were approximately the same, and the overall forms of the distributions were very similar for 4 of the 5 subjects. For Rat 13, the mode of the drinking distribution occurred later in the interval than did running, although the starting times were approximately the same.

Figure 10 depicts the temporal distributions of drinking and running in the two conditions of Phase 9, plotted together for ease of comparison. In Phase 9, either the drinking tube (in Phase 9a) was retracted or the running wheel (in Phase 9b) was locked for the entire session. Running and drinking started at approximately the same time within their respective IRIs in both conditions for each subject. The actual heights of the distributions varied considerably, as did the session totals for each (cf. Table 4). Rat 13 became ill and died during this phase, so its panel represents the average of only four (rather than eight) sessions. Rat 11 was able to contact the drinking tube occasionally even when it was retracted, so its data are not depicted.

Table 5 contains the percentages of each type of IRI for each phase and each subject. The interval types were defined as in Experiment 1: (a) intervals in which drinking preceded running, (b) intervals containing running preceding drinking, (c) intervals containing drinking but no running, (d) intervals containing running but no drinking, and (e) intervals containing neither activity.

The percentage of intervals containing both drinking and running was low (less than 25\%) for 4 of the 5 subjects in Phase 3, in which subjects had free access to both activities. Rat 11 was the only exception, with $60.8 \%$ of the intervals containing both activities. For all subjects, drink-run intervals occurred more frequently than run-drink intervals. In all subsequent conditions requiring a single lever press for access to the drinking tube or the 


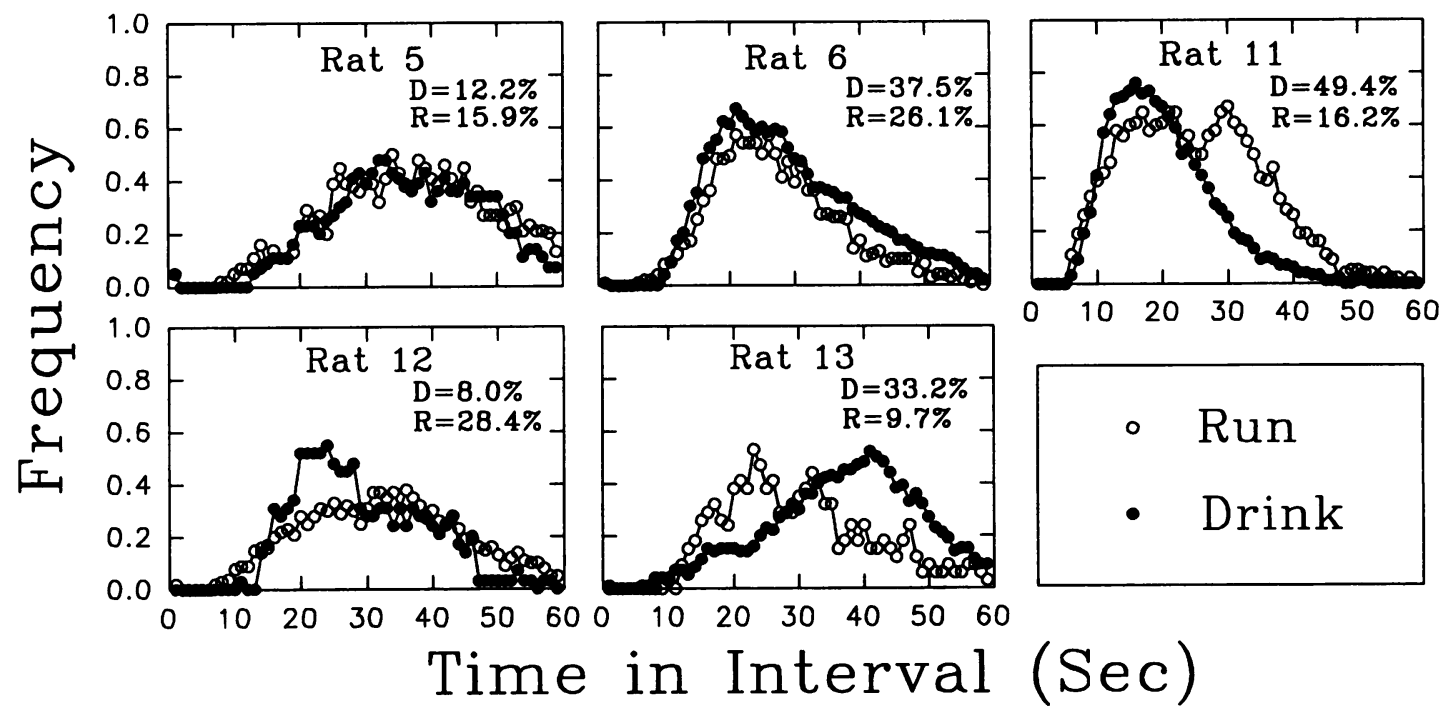

Fig. 9. Temporal frequency distributions of drinking and running that occurred in drinking-only and runningonly intervals for each subject in the exclusive choice condition of Phase 7 of Experiment 2. The drinking and running distributions are plotted together even though they occurred in separate IRIs in order to compare their temporal characteristics. The numbers in each panel represent the obtained percentages of drinking-only and running-only intervals.

running wheel, the percentage of intervals containing both activities decreased dramatically to $11 \%$ or less with all subjects, even with Rat 11. When subjects were shifted to the exclusive choice condition (Phase 7), the number of in- tervals containing drinking increased for 3 of the 4 subjects. The number of intervals containing running decreased for all subjects, but the decrease was sometimes small.

In Phase 9, either the drinking tube was

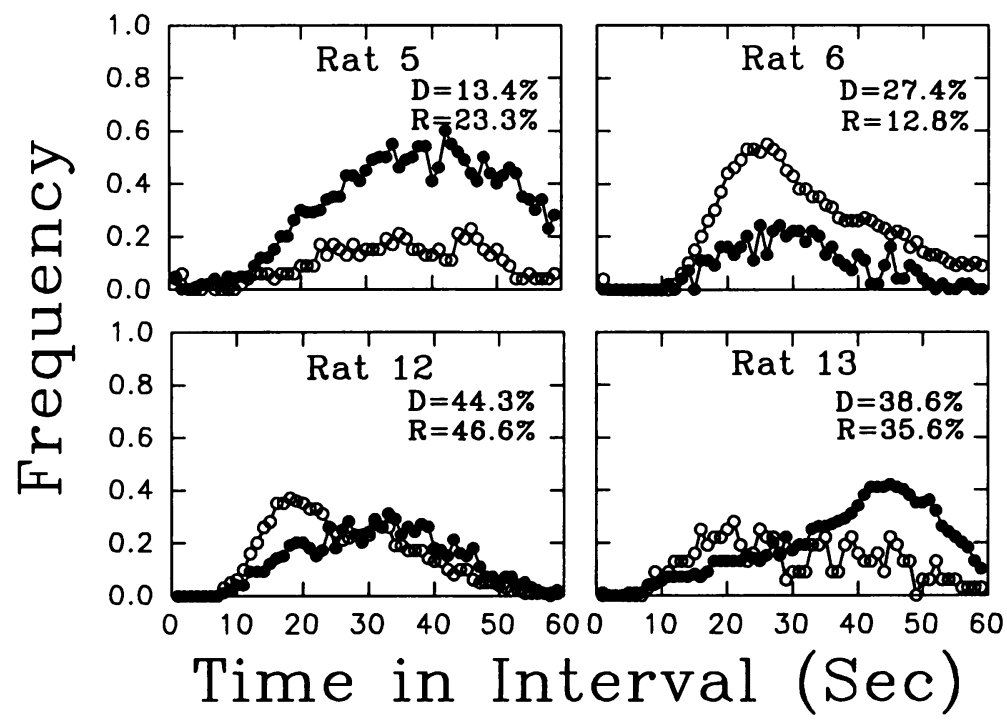

Fig. 10. Temporal frequency distributions of drinking and running that occurred in drinking-only and runningonly intervals for each subject in the no-choice condition of Phase 9 of Experiment 2. The drinking and running distributions are plotted together even though they occurred in separate sessions in order to compare their temporal characteristics. Rat 11 was able to contact the drinking tube occasionally even when it was withdrawn, so its data are not plotted. Filled circles represent drinking, and open circles represent running. 
Table 5

Percentage of each type of interval observed in each phase of Experiment 2.

\begin{tabular}{|c|c|c|c|c|c|c|}
\hline Condition & Interval type & Rat 5 & Rat 6 & Rat 11 & Rat 12 & Rat 13 \\
\hline $\begin{array}{l}\text { Phase } 3 \\
\text { free access }\end{array}$ & $\begin{array}{l}\text { Drink } \rightarrow \text { run } \\
\text { Run } \rightarrow \text { drink } \\
\text { Drink only } \\
\text { Run only } \\
\text { No run or drink }\end{array}$ & $\begin{array}{r}17.9 \\
3.1 \\
12.5 \\
20.2 \\
46.3\end{array}$ & $\begin{array}{r}12.8 \\
0.6 \\
12.5 \\
30.7 \\
43.5\end{array}$ & $\begin{array}{r}59.7 \\
1.1 \\
18.2 \\
16.5 \\
4.5\end{array}$ & $\begin{array}{r}21.9 \\
1.4 \\
4.5 \\
53.7 \\
18.5\end{array}$ & $\begin{array}{r}9.7 \\
0.6 \\
26.1 \\
13.4 \\
50.3\end{array}$ \\
\hline $\begin{array}{l}\text { Phase } 5 \\
\text { recovery of Phase } 3\end{array}$ & $\begin{array}{l}\text { Drink } \rightarrow \text { run } \\
\text { Run } \rightarrow \text { drink } \\
\text { Drink only } \\
\text { Run only } \\
\text { No run or drink }\end{array}$ & $\begin{array}{r}0.6 \\
1.1 \\
14.5 \\
15.9 \\
67.9\end{array}$ & $\begin{array}{r}6.5 \\
0.9 \\
25.9 \\
27.0 \\
39.8\end{array}$ & $\begin{array}{r}56.5 \\
1.7 \\
23.0 \\
12.2 \\
6.5\end{array}$ & $\begin{array}{r}12.5 \\
1.4 \\
4.8 \\
59.1 \\
22.2\end{array}$ & $\begin{array}{r}3.4 \\
0.3 \\
31.3 \\
13.1 \\
52.0\end{array}$ \\
\hline $\begin{array}{l}\text { Phase } 6 \\
\text { FR } 1 \text { each activity }\end{array}$ & $\begin{array}{l}\text { Drink } \rightarrow \text { run } \\
\text { Run } \rightarrow \text { drink } \\
\text { Drink only } \\
\text { Run only } \\
\text { No run or drink }\end{array}$ & $\begin{array}{r}0.6 \\
0.0 \\
11.6 \\
29.8 \\
58.0\end{array}$ & $\begin{array}{r}4.2 \\
0.0 \\
25.3 \\
28.6 \\
41.9\end{array}$ & $\begin{array}{r}10.7 \\
0.3 \\
31.8 \\
33.4 \\
23.7\end{array}$ & $\begin{array}{r}3.1 \\
0.3 \\
4.3 \\
39.5 \\
52.8\end{array}$ & $\begin{array}{r}3.1 \\
1.1 \\
48.0 \\
17.0 \\
30.7\end{array}$ \\
\hline $\begin{array}{l}\text { Phase } 7 \\
\text { exclusive choice }\end{array}$ & $\begin{array}{l}\text { Drink } \rightarrow \text { run } \\
\text { Run } \rightarrow \text { drink } \\
\text { Drink only } \\
\text { Run only } \\
\text { No run or drink }\end{array}$ & $\begin{array}{r}0.0 \\
0.0 \\
12.2 \\
16.2 \\
71.6\end{array}$ & $\begin{array}{r}0.0 \\
0.0 \\
37.5 \\
26.1 \\
36.4\end{array}$ & $\begin{array}{l}- \\
- \\
-\end{array}$ & $\begin{array}{r}0.0 \\
0.0 \\
8.0 \\
28.7 \\
63.3\end{array}$ & $\begin{array}{r}0.0 \\
0.0 \\
33.2 \\
9.7 \\
57.1\end{array}$ \\
\hline $\begin{array}{l}\text { Phase } 8 \\
\text { recovery of Phase } 6\end{array}$ & $\begin{array}{l}\text { Drink } \rightarrow \text { run } \\
\text { Run } \rightarrow \text { drink } \\
\text { Drink only } \\
\text { Run only } \\
\text { No run or drink }\end{array}$ & $\begin{array}{r}0.0 \\
0.3 \\
11.1 \\
6.5 \\
82.1\end{array}$ & $\begin{array}{r}0.9 \\
0.2 \\
19.3 \\
11.6 \\
67.9\end{array}$ & $\begin{array}{r}0.0 \\
0.0 \\
21.9 \\
16.5 \\
61.6\end{array}$ & $\begin{array}{r}10.5 \\
0.0 \\
13.6 \\
29.3 \\
46.6\end{array}$ & $\begin{array}{r}0.6 \\
1.4 \\
49.7 \\
6.8 \\
41.5\end{array}$ \\
\hline $\begin{array}{l}\text { Phase } 9 \mathrm{a} \\
\text { FR } 1 \text { no choice, no water }\end{array}$ & $\begin{array}{l}\text { Drink } \rightarrow \text { run } \\
\text { Run } \rightarrow \text { drink } \\
\text { Drink only } \\
\text { Run only } \\
\text { No run or drink }\end{array}$ & $\begin{array}{r}0.0 \\
0.0 \\
0.0 \\
23.3 \\
76.7\end{array}$ & $\begin{array}{r}0.0 \\
0.0 \\
0.0 \\
12.8 \\
87.2\end{array}$ & $\begin{array}{r}0.0 \\
0.0 \\
0.0 \\
44.9 \\
55.1\end{array}$ & $\begin{array}{r}0.0 \\
0.0 \\
0.0 \\
46.6 \\
53.4\end{array}$ & $\begin{array}{l}- \\
- \\
-\end{array}$ \\
\hline $\begin{array}{l}\text { Phase } 9 \mathrm{~b} \\
\text { FR } 1 \text { no choice, no wheel }\end{array}$ & $\begin{array}{l}\text { Drink } \rightarrow \text { run } \\
\text { Run } \rightarrow \text { drink } \\
\text { Drink only } \\
\text { Run only } \\
\text { No run or drink }\end{array}$ & $\begin{array}{r}0.0 \\
0.0 \\
13.4 \\
0.0 \\
86.6\end{array}$ & $\begin{array}{r}0.0 \\
0.0 \\
27.4 \\
0.0 \\
72.2\end{array}$ & $\begin{array}{r}0.0 \\
0.0 \\
66.8 \\
0.0 \\
33.2\end{array}$ & $\begin{array}{r}0.0 \\
0.0 \\
44.3 \\
0.0 \\
55.7\end{array}$ & $\begin{array}{r}0.0 \\
0.0 \\
38.6 \\
0.0 \\
61.4\end{array}$ \\
\hline $\begin{array}{l}\text { Phase } 10 \\
\text { recovery of Phase } 3\end{array}$ & $\begin{array}{l}\text { Drink } \rightarrow \text { run } \\
\text { Run } \rightarrow \text { drink } \\
\text { Drink only } \\
\text { Run only } \\
\text { No run or drink }\end{array}$ & $\begin{array}{r}0.3 \\
0.6 \\
9.1 \\
19.9 \\
70.2\end{array}$ & $\begin{array}{r}0.6 \\
0.0 \\
18.5 \\
9.9 \\
71.0\end{array}$ & $\begin{array}{r}3.4 \\
0.3 \\
37.2 \\
30.1 \\
29.0\end{array}$ & $\begin{array}{r}23.9 \\
2.6 \\
7.7 \\
46.3 \\
19.6\end{array}$ & $\begin{array}{l}- \\
- \\
-\end{array}$ \\
\hline
\end{tabular}

Note. Rat 11 was able to reach the drinking tube even when it was retracted during Phase 7 , exclusive choice, allowing some intervals to contain both drinking and running. Therefore, those data are not included. Rat 13 died during the no water condition of Phase 9, so data are not available for that condition or for Phase 10.

retracted or the running wheel was locked for the entire session. The data in this condition can be compared to the previous phase, which had the same lever-press requirement and recovered the drinking and running levels of Phase 6 . When the drinking tube was not available, the percentage of intervals containing running (running only) increased in all subjects from that obtained when both activities were available. When the running wheel was locked, the percentage of intervals containing drinking (drinking only) increased in 4 of the 5 subjects.

As in Experiment 1, these results cast doubt on the notion that periodic food schedules directly modulate the tendencies to engage in drinking and running at different points in the IRI. Drinking and running usually occupied approximately the same portions of the IRI in the three conditions of free access, exclusive 
choice, and no choice. Their onset times were equivalent. For 4 of 5 rats, the drink-run pattern occurred infrequently. Rat 11 was the exception in Phase 3, but this frequency dropped to less than $4 \%$ in Phase 10, which was the final exposure to the conditions of Phase 3.

\section{EXPERIMENT 3}

The periodic food schedules in Experiments 1 and 2 were restricted to an FT 60-s schedule. In schedules with longer IRIs, such as an FT 240 -s schedule, more time is available within each interval for the allocation of both drinking and running. It is possible that these longer intervals would entrain drinking and running to occur at different periods, and more variation would occur in the behavioral sequences. Experiment 3 compared the activity distributions obtained on an FT 60-s schedule with those obtained on an FT 240-s schedule in an ABAB design.

\section{METHOD}

\section{Subjects and Apparatus}

Four naive female albino rats, purchased from Charles River, were housed individually in one room with 24-hr light. Each rat was approximately 6 months old at the beginning of the study. Their weights were reduced to $80 \%$ of their free-feeding weights by restricting food intake over a period of 10 days. Water was freely available in the home cages.

The apparatus was the same as that used in Experiments 1 and 2.

\section{Procedure}

All subjects were exposed to four experimental conditions in an $\mathrm{ABAB}$ design.

Condition A: FT 60 s free access. Food pellets were delivered on an FT 60 -s schedule, and subjects had free and continuous access to the drinking tube, running wheel, and the oak block. Lever presses had no programmed consequences. All sessions began with a pellet delivery and lasted $45 \mathrm{~min}$. The drinking tube, chewing block, and running wheel were presented to each subject at the beginning of the session with the first pellet delivery, and were retracted or locked at the end of the 45th interval. This condition was in effect until the session totals and the temporal distributions of all activities within the IRI appeared stable for at least eight sessions for each subject. Subjects were exposed to this condition for 54 sessions in the first exposure and 20 sessions in the second exposure.

Condition B: FT 240 s free access. Food pellets were delivered on an FT 240-s schedule. Sessions lasted $80 \mathrm{~min}$, with the completion of the 20th interfood interval. This condition was similar to Condition A in all other respects. Subjects were exposed to this condition for 27 sessions in the first exposure and 20 sessions in the second exposure.

\section{Results AND Discussion}

The session totals and averaged temporal distributions of each activity for each subject in the second exposure to Condition $A$ and Condition B accurately replicated the results obtained during their first exposure. Data are reported for the last four sessions in each exposure to Condition A, yielding averages over eight sessions. Because Condition B contained only 20 interfood intervals per session, data are reported for the last six sessions in each exposure, yielding averages over 12 sessions.

In Condition A, the temporal distributions of drinking and running in each interval type were very similar to those observed in Phase 5 of Experiments 1 and 2. Only data from a representative subject, Rat 22 , are shown in Figure 11. As before, the drink-run sequence was observed in less than $20 \%$ of the intervals. Over $65 \%$ of the IRIs contained running without drinking.

Figure 12 depicts the temporal distributions of drinking (in drinking-only intervals) and running (in running-only intervals), plotted together for ease of comparison. Rats 22 and 23 showed the now familiar similarity in the temporal characteristics of the two activities. Rat 24 showed a novel distribution of drinking, but it was the result of only two drinking-only intervals in the eight sessions. It is important to remember that the height of the distributions is not indicative of the absolute level of the activity at that second, because the divisor for the ordinate was the number of times that interval type occurred. The unique drinking distribution of Rat 24 is simply the result of very few occurrences of drinking-only intervals. Rat 21 was unique in that drinking occurred very late in the IRI in those few intervals (10 intervals or $2.8 \%$ ) when it occurred alone. Nevertheless, the drinking distribution 


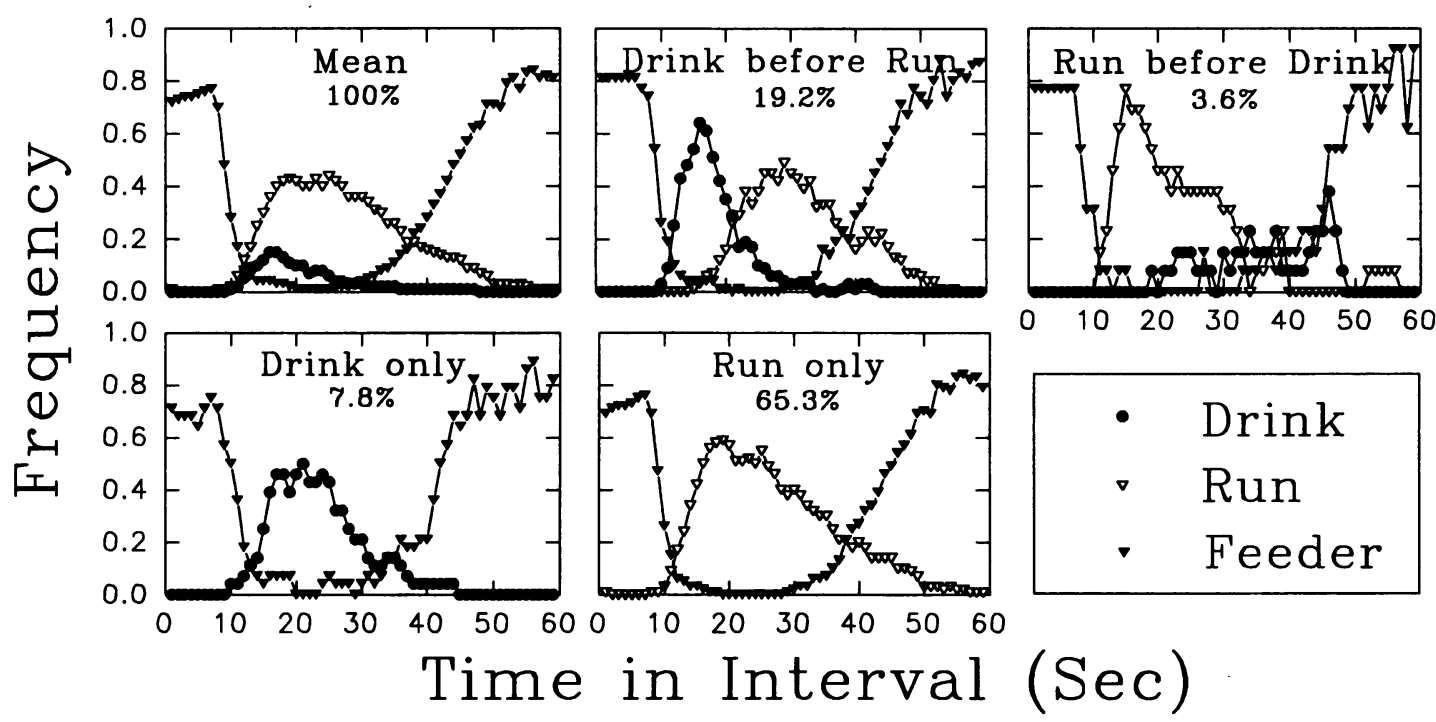

Fig. 11. Temporal frequency distributions of activities for Rat 22 occurring on an FT 60-s schedule in Condition A of Experiment 3. See text for a description of interval types. The number in each panel represents the percentage of that interval type observed in this condition.

of Rat 21 is an accurate indicator of the temporal position of drinking.

In Condition B, an FT 240-s food schedule was in effect. This lean schedule produced substantially lower amounts of drinking in all rats than had the FT 60-s schedule. The temporal frequency distributions of drinking and running for each subject were very similar, with no systematic differences between subjects for any of the interval types. Data from the same representative subject, Rat 22, are shown in Figure 13 for each type of interval. The tem-

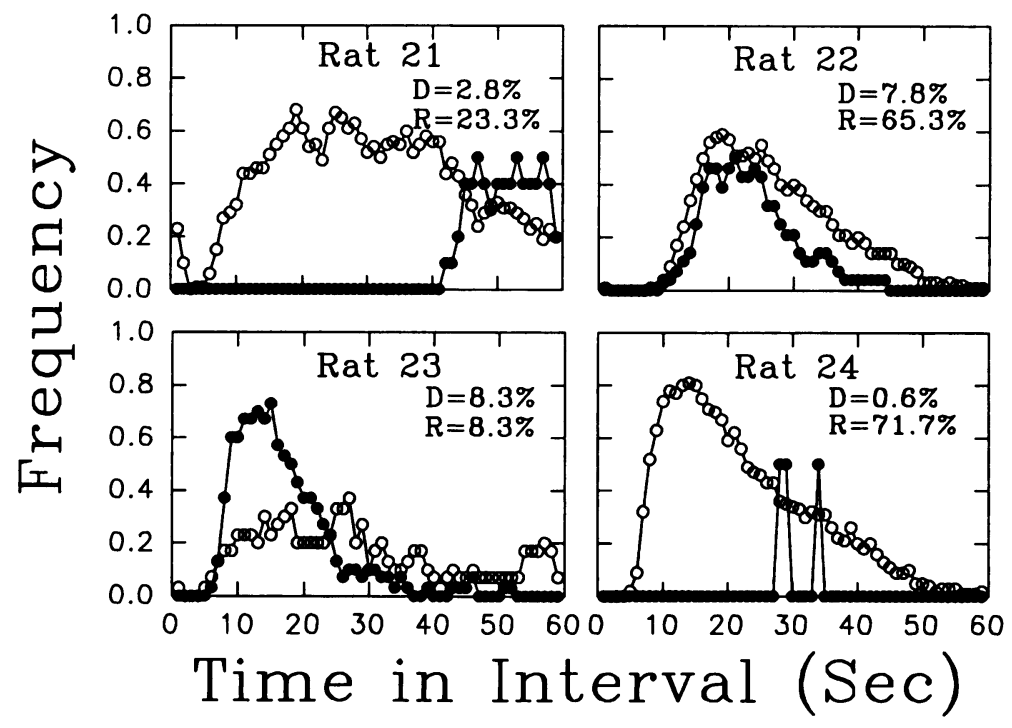

Fig. 12. Temporal frequency distributions of drinking and running that occurred in drinking-only and runningonly intervals for each subject on an FT 60-s schedule in Condition A of Experiment 3. The drinking and running distributions are plotted together even though they occurred in separate IRIs in order to compare their temporal characteristics. The numbers in each panel represent the obtained percentages of drinking-only and running-only intervals. Filled circles represent drinking, and open circles represent running. 

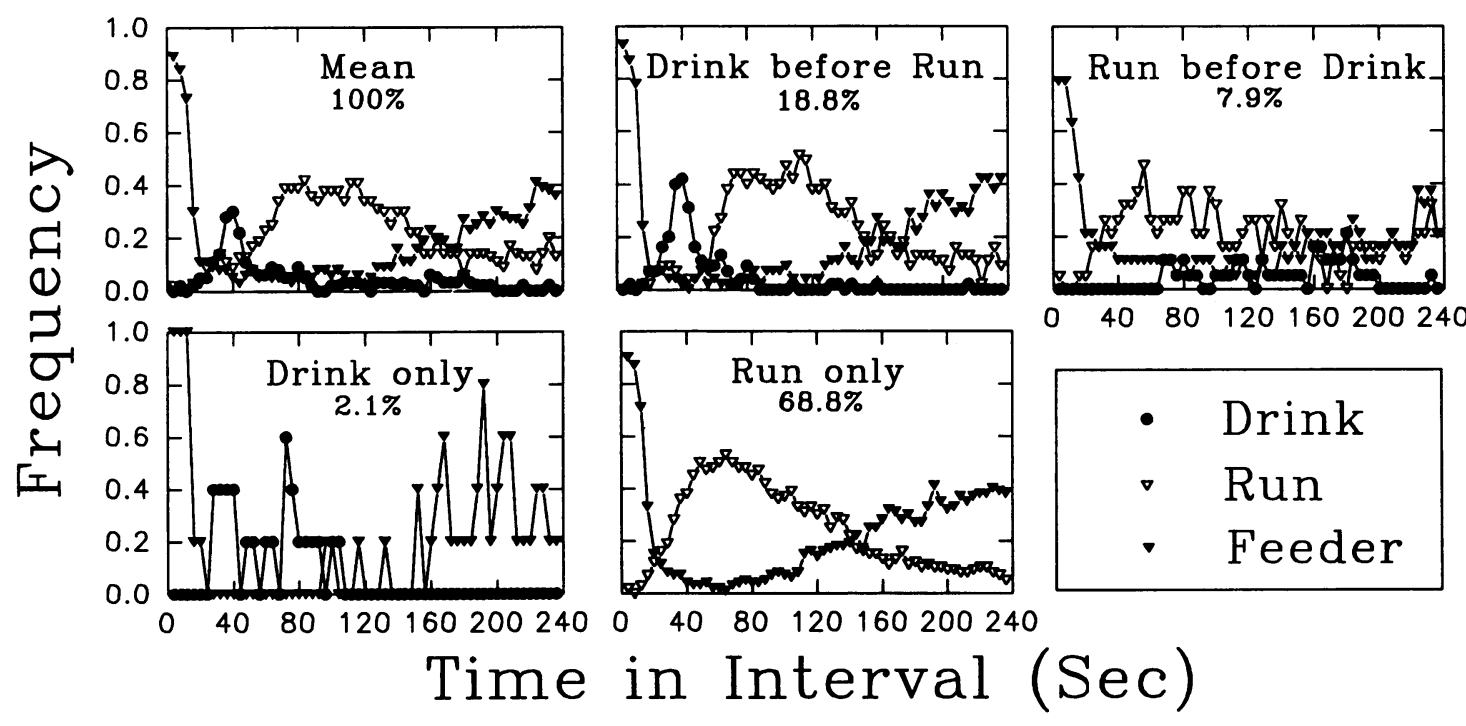

Fig. 13. Temporal frequency distributions of activities for Rat 22 occurring on an FT 240-s schedule in Condition B of Experiment 3. See text for a description of the interval types. The number in each panel represents the percentage of that interval type observed in this condition.

poral sequence of drinking and running over the IRI on the FT 240-s schedule was very similar to that obtained on the FT 60 -s schedule (cf. Figure 11). It is especially interesting to note that the onset of drinking in drinkbefore-run intervals occurred at the same time in the IRI as the onset of running in runbefore-drink intervals, and this latter interval type occurred much more frequently in the FT 240-s schedule than in the FT 60 -s schedule (15\% vs. $2 \%$ ). This similarity in onset times was evident in all subjects.

Table 6 contains the percentages of each interval type obtained from both conditions. In the FT 240-s schedule, all subjects increased the percentage of intervals containing both drinking and running over the level obtained on the FT 60-s schedule, and much of the increase was due to a larger percentage of the run-drink intervals. Only 2 subjects increased the percentage of the drink-run intervals. The percentage of drinking-only intervals was very low and was approximately the same in both conditions for all subjects. The percentage of running-only intervals was not systematically affected by the change to the FT 240-s schedule; the percentage increased with 2 subjects, decreased with 1 and remained approximately the same for another. The percentage of intervals containing neither drinking nor running was very low for all subjects. The 2 sub- jects that had high percentages of no-run-ordrink intervals under the FT 60 -s schedule, Rat 21 (54\%) and Rat 23 (72\%), reduced their percentages of this interval type substantially during the FT 240-s schedule (to about 3\% for both subjects).

A comparison of the temporal distributions of drinking and running occurring in drinkingonly and running-only intervals is more difficult with the FT 240-s schedule than with the FT 60-s schedule because drinking-only intervals occurred so infrequently. Although the percentage of drinking-only intervals was quite low in both conditions, there were only 20 intervals per session on the FT 240-s sched-

Table 6

Percentage of each type of interval observed in each phase of Experiment 3.

\begin{tabular}{llrrrr}
\hline $\begin{array}{c}\text { Con- } \\
\text { dition }\end{array}$ & Interval type & $\begin{array}{c}\text { Rat } \\
21\end{array}$ & $\begin{array}{c}\text { Rat } \\
22\end{array}$ & $\begin{array}{c}\text { Rat } \\
23\end{array}$ & $\begin{array}{c}\text { Rat } \\
24\end{array}$ \\
\hline \hline FT 60s & Drink $\rightarrow$ run & 16.9 & 19.2 & 10.8 & 26.9 \\
& Run $\rightarrow$ drink & 3.6 & 3.6 & 0.6 & 0.6 \\
& Drink only & 2.8 & 7.8 & 8.3 & 0.6 \\
& Run only & 23.3 & 65.3 & 8.3 & 71.7 \\
& No run or drink & 53.3 & 4.2 & 71.9 & 0.3 \\
FT 240 s & Drink $\rightarrow$ run & 24.2 & 18.8 & 47.5 & 26.3 \\
& Run $\rightarrow$ drink & 9.6 & 7.9 & 15.4 & 26.7 \\
& Drink only & 1.7 & 2.1 & 13.8 & 0.4 \\
& Run only & 61.3 & 68.8 & 20.4 & 46.3 \\
& No run or drink & 3.2 & 2.4 & 2.9 & 0.3 \\
\hline
\end{tabular}




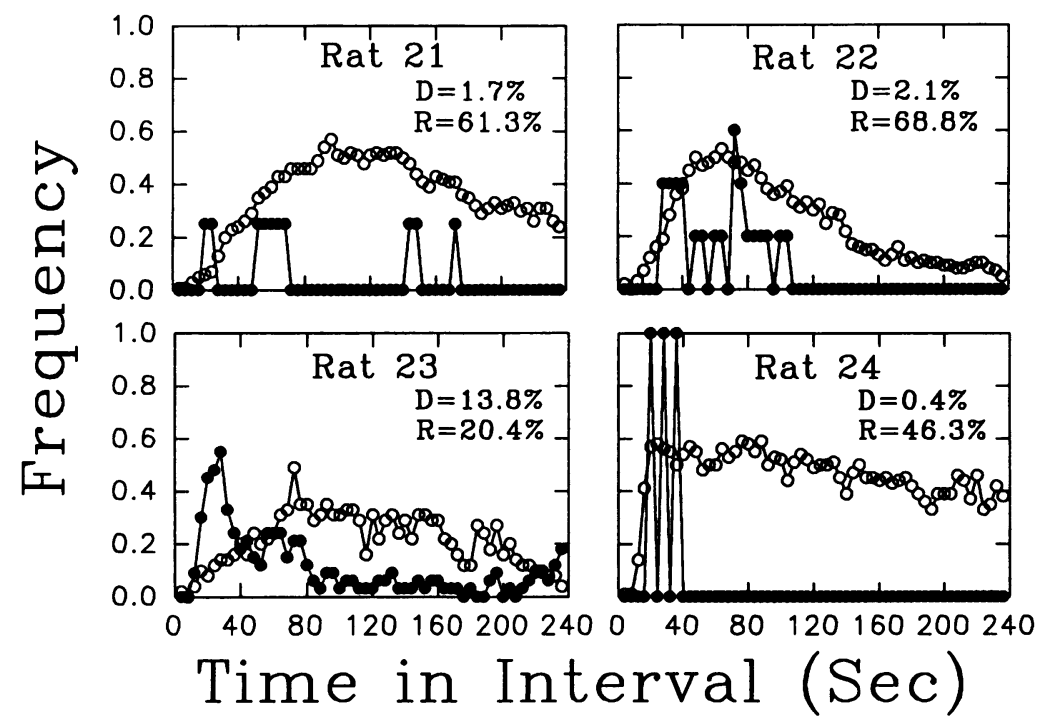

Fig. 14. Temporal frequency distributions of drinking and running that occurred in drinking-only and runningonly intervals for each subject on an FT 240-s schedule in Condition B of Experiment 3. The drinking and running distributions are plotted together even though they occurred in separate IRIs in order to compare their temporal characteristics. The numbers in each panel represent the obtained percentages of drinking-only and running-only intervals. Filled circles represent drinking, and open circles represent running.

ule, compared to 45 intervals per session for the FT 60-s schedule. The obtained drinking distributions in 1 subject resulted from as few as three intervals over 12 sessions. Figure 14 depicts these drinking and running distributions obtained from drinking-only and running-only intervals. Even though comparison of the shapes of the distributions is not appropriate in this condition because of the large difference in the number of intervals represented by them, the drinking and running distributions did overlap in all subjects. This overlap implies that drinking and running did not occupy different portions of IRIs when the activities occurred alone in the FT 240-s schedule.

Statistical analysis of the distributions of the starting times of drinking and running was not informative for 3 of the 4 subjects because of the very small number of drinking-only intervals. The frequency of these intervals for Rat 23, however, did permit statistical analysis. This analysis revealed that the drinking and running distributions could be described as representing independent samples from the same hypothetical distribution with at least $95 \%$ certainty, $t(58)=2.3, p<.05$. This similarity demonstrates that drinking and running began at the same point within the IRI in drinking-only and running-only intervals for this subject.

\section{GENERAL DISCUSSION}

Much of the research related to temporal control on periodic reward schedules and to theories of schedule induction has emphasized the temporal regularities and behavioral stereotypy that occur within IRIs. Explanations of this temporal regularity have often hypothesized a modulating influence of the schedule on the motivational states of the animal. In the simplest case, models of schedule induction have posited a facilitatory relation between properties of periodic food schedules and adjunctive drinking or other schedule-induced activities (Cohen et al., 1985; Falk, 1966, 1971; Freed, Zec, \& Mendelson, 1977; Killeen, 1975, 1979; Rosellini, 1985). Some theorists have extended the idea (that periodic schedules directly modulate the motivational states of animals) to more than one activity to explain the frequently obtained multimodal activity distributions occurring within IRIs (e.g., Cohen et al., 1985; Staddon, 1977; Thompson \& Lubinski, 1986). Our results do not support this extension to a sequence of activities forming multimodal distributions. 
One purpose of the present study was to isolate the determinants of these multimodal distributions. Our results indicated that the distributions obtained by averaging across all IRIs in a session do not accurately represent the transitions between activities that occur within most IRIs. We obtained multimodal distributions in Experiment 1 (Figure 5) when we averaged across all intervals, but these multimodal distributions were obtained by averaging across intervals with dissimilar activity transitions. Drinking and running most often occurred in separate intervals, with approximately equal starting times but with variation in bout duration. These averaged multimodal distributions have been the basis on which the previously cited theorists have proposed that periodic schedules modulate the animal's motivational states, causing activities to occur at particular periods within the IRI. Our results lead us to conclude that transitions between activities within individual IRIs in rats do not result from a direct influence by the schedule on the sequence of tendencies to drink and run.

First, a direct influence of the periodic schedule on the tendency to drink or run would imply that these activities should occur at different periods within the IRI. All three experiments demonstrated that when drinking and running occurred in separate intervals, their onset times were approximately equal. The similarity in the distributions of drinking and running was evident (a) when rats had free access to each activity (Experiment 1, Phase 5), (b) when a single lever press was required for access to each activity (Experiment 2, Phase 6), (c) when subjects could select only a single activity within an interval (Experiment 2, Phase 7), (d) when the availability of the drinking tube or running wheel was manipulated by the experimenter independently of the rat's behavior (Experiment 2, Phase 9), and (e) when the duration of the IRI was extended to 240 s (Experiment 3, Condition B).

Second, the drink-run sequence was usually observed in only a small percentage (less than $20 \%$ ) of the intervals. Rat 1 in Phase 3 of Experiment 1 and Rat 11 in Phase 3 of Experiment 2 were exceptions, but their drinkrun pattern was reduced in subsequent conditions. This pattern did not recover during subsequent replications of the free access condition. Rather, drinking and running usually occurred in separate intervals.
Finally, when the duration of the IRI was changed from 60 to $240 \mathrm{~s}$ in Experiment 3, the increase in the number of intervals containing both drinking and running in the FT 240-s schedule was due largely to an increase in the number of intervals in which running preceded drinking, rather than the reverse. This sequence of activities should not occur if the schedule directly modulates motivational states in the same manner in every interval.

What evidence supports the hypothesis of a modulating influence of the schedule? If this influence is discounted, what other mechanisms might be responsible? It is useful to separate two related types of evidence: evidence that directly points toward a modulating influence and evidence that indicates a low degree of substitutability between drinking and running. A modulating influence is supported by the observation that the drink-run sequence did occur in some intervals. In the context of these experiments, 14 rats were exposed to the FT 60-s free access condition, and for 12 of the rats, the drink-run pattern occurred in less than $20 \%$ of the intervals in all conditions. The occasional transition from drinking to running (or vice versa) observed in most subjects should not imply a direct influence by the schedule. The drink-run sequence occurred substantially more frequently than the run-drink sequence. Why might the drink-run sequence be more likely? It is easy to postulate a possible mechanism for the difference without assuming that the periodic schedule modulates motivational states. If preferred drinking bouts are shorter in duration than are running bouts (as was found in Experiment 1), then the offset of drinking in drink-run sequences would normally occur earlier in the IRI than would the offset of running in run-drink sequences. As time in the interval progresses, the dominant food-related behavior becomes increasingly likely. Thus, food-related behavior should be a stronger competitor after a running bout has ended (preventing drinking from occurring) than after a drinking bout has ended. This hypothesis is supported by the finding in Experiment 3 of an increase in the number of run-drink intervals when rats were shifted to the longer FT 240-s schedule.

The poor substitutability between drinking and running remains unexplained. Drinking and running appeared to be slightly substitutable for each other but only in certain situations. For example, in the fixed-ratio sched- 
ule conditions of Experiment 1, running levels did not increase as drinking levels decreased, and the temporal position of running did not shift to earlier parts in the interval previously occupied by drinking. This lack of substitutability within the IRI has often been explained by assuming that periodic schedules modulate motivational states to preclude substitution. In most conditions in each experiment, however, running clearly did occupy the same temporal position as drinking. Running and drinking often began at the same postfood period, and their temporal distributions were often strikingly similar. Thus, the poor substitutability was not due to any direct influence of the periodic schedule on the underlying motivational systems. Instead, it is more likely related to the mechanisms responsible for the preference for drinking after food delivery.

Substitutability was greater in other conditions. For example, in the exclusive choice condition of Experiment 2, drinking increased and running decreased in all subjects compared to the free access condition. In the no choice condition, drinking levels were higher when running was not available, and running levels were higher when drinking was not available. Each finding implies either a degree of substitutability or competition for available time. The total amount of time engaged in drinking and running in intervals containing both activities was not significantly longer than the time spent running when it occurred alone. The total amount of time engaged in activities unrelated to obtaining food appeared to be roughly constant across intervals, with running and drinking substituting for one another or competing with one another for expression. This substitution, or competition, has been widely observed in other studies that have demonstrated that the addition of a running wheel suppresses the time spent drinking (e.g., Experiment 2; Riley, Peele, Richard, \& Kulkosky, 1981; Segal, 1969; Staddon \& Ayres, 1975; Wetherington \& Riley, 1986).

The emphasis in most studies concerned with the temporal organization of behavior within the IRI has been placed on the peaks, or modes, of the averaged frequency distributions, such as those in the first column of Figure 5 (cf. Reid \& Staddon, 1990; Staddon, 1977). It is important not to confuse a positive slope in such a distribution with an individual rat's increased tendency to engage in that activity.
The peaks of these averaged distributions are functions of variables with values that are unique to each activity, making peaks very poor indicators of motivational states. For example, preferred bout lengths for drinking and running are typically not of the same duration (cf. Experiment 1). The activity with the shorter bout lengths will usually have a shorter, more pointed distribution, as is often seen with drinking. In addition, the preferred rates of licking and running are not equal. The measured rate of running in a wheel depends critically upon the size of the wheel and the number of "counts" per revolution, as well as the motivational state of the subject. Drinking rate depends more on the physical characteristics of licking. The activity with the higher rate will probably have a more pointed distribution. Finally, the times required to reach the preferred rates of licking and running are probably substantially different. Licking occurs at a relatively fixed rate that is reached only a second or two after licking begins. Running typically requires accelerating a wheel with substantial inertia, and rats often change the direction of the wheel's rotation.

We place our emphasis on the time of an activity's onset within the IRI rather than the distribution's peak because it indicates the time of the transition from one activity to another. If periodic food schedules can modulate the subject's motivational states to cause transitions between activities at relatively fixed points within the IRI, the time of an activity's onset is a more accurate indicator of this point.

Many studies have demonstrated multimodal activity patterns in rats on a wide range of interval durations (see review in Reid \& Staddon, 1990). The multimodal pattern that has most often been reported is drink then run, as in this study. Do the data in these studies contradict our conclusions and argue in favor of a modulating influence of periodic schedules? We found that other published data actually support our conclusions. For example, although Figure 2 (Rat 4) from Staddon and Ayres (1975) has often been republished as the classic example of the drink-run pattern, the running and drinking distributions overlapped substantially in 4 of their 5 rats. Importantly, running and drinking started at approximately the same time within the interval. Overall, there was much variation in the sequence of the activities. The study by Reid and Staddon 
(1990) also contains an example of the drinkrun pattern. Their Figure 8.1 was an early analysis of Rat 1 in Phase 3 of Experiment 1 of this study, so it does not represent an additional example of a stereotyped behavior pattern. As pointed out above, Rat 1 demonstrated this drink-run pattern in that condition substantially more than did the other 4 rats, but the pattern was transient. Other studies demonstrating multimodal activity patterns in rats show substantial (often complete) overlap of their distributions, even though their respective modes occur at different points in the interval (Killeen, 1975; Knutson \& Schrader, 1975; Lucas et al., 1988; McIntire, Lundervold, Calmes, Jones, \& Allard, 1983; Reid et al., 1985; Roper, 1978; Wetherington \& Riley, 1986). To our knowledge, no other data that offer a detailed examination of the intervals that make up these averaged distributions are available.

Based on several observations, we conclude that the most frequent sequence of activities on periodic food schedules is a single period of drinking, running, or almost any other activity (schedule induced or not), beginning soon after consuming the pellet, followed by returning to the feeder in anticipation of the next pellet delivery. Published distributions show the same overlap in distributions that our results show; our results demonstrate that overlapping multimodal distributions are the result of averaging across typically unimodal intervals; the distributions of the times of onset of drinking and running were the same in this study as well as in that of Staddon and Ayres (1975); and IRIs contain substantial variation in the order of activities when two or more activities occur, especially with long IRIs. Most rats do not show a stereotyped drink-run sequence within most IRIs.

\section{Mechanisms of Temporal Organization}

Many studies have reported schedule-induced behavior with unimodal distributions in rats (Edwards \& Roper, 1982; Killeen, 1975; King, 1974; Reberg, Mann, \& Innis, 1977; Reid \& Dale, 1983; Reid \& Staddon, 1982; Wetherington \& Riley, 1986). In their formal analysis of the temporal distributions of drinking, Reid and Dale (1983) demonstrated that periodic food schedules directly facilitate foodrelated activities, and temporal distributions of schedule-induced drinking were determined by the temporal distributions of the dominant food-related activities. They demonstrated that at each second in the IRI, increases (or decreases) in the distribution of food-related activities (head-in-feeder) corresponded very closely to decreases (or increases) in the drinking distributions. Their result would be expected (and trivial) if drinking and food-related activities were mutually exclusive and exhaustive, but in their experiments much time remained available for other activities. Reid et al. (1985) demonstrated the same reciprocal relation with activities that were not schedule induced, but were produced by rats on a periodic water schedule. All activities had essentially the same temporal distributions in the interval and complemented the distribution of reward-related activities.

Hence, reward and temporal control determine the temporal distributions of reward-related activities, which, in turn, determine the unimodal distributions of induced and noninduced activities. In simple periodic schedules, time engaged in reward-related activities (consuming, working, or looking for reward) determines the time available for all other activities, which probably have only a small impact on the amount of reward-related activities (see review in Reid \& Staddon, 1990).

It is now clear that the temporal characteristics of reward-related activities play an important role in the temporal organization of essentially all types of behavior of periodic reward schedules. Following Timberlake and his colleagues (Lucas et al., 1988; Timberlake, 1990; Timberlake \& Lucas, 1989) we have used the term focal search to describe these reward-related activities in order to emphasize the functional role they serve in the rat's natural environment as it forages for food. Focal search corresponds roughly to terminal behaviors in Staddon's (1977) model of schedule induction. In periodic schedules, focal search comes under temporal control and is the dominant, highest priority activity during the beginning and end of the IRIs when the probability of finding food is high (Reid \& Dale, 1983).

The unimodal distributions of drinking and running in the present experiments are entirely consistent with the notion that unimodal distributions on periodic reward schedules are determined by the temporal distribution of focal search. The determinants of the temporal 
characteristics of the distributions appear to be the same for schedule-induced activities as well as for noninduced activities. Even occasional drink-run and run-drink sequences are consistent with Reid and Dale's (1983) conclusion that the temporal organization of schedule-induced drinking, running, and other activities occurring within the IRI is determined by the temporal distribution of focal search, which is facilitated by and under temporal control of the periodic food schedule.

\section{REFERENCES}

Alferink, L. A., Bartness, T. J., \& Harder, S. (1980). Control of the location of polydipsic licking in the rat. Journal of the Experimental Analysis of Behavior, 33, 119-129.

Cohen, P. S., Looney, T. A., Campagnoni, F. R., \& Lawler, C. P. (1985). A two-state model of reinforcerinduced motivation. In F. R. Brush \& J. B. Overmeir (Eds.), Affect, conditioning, and cognition: Essays on the determinants of behavior (pp. 281-297). Hillsdale, NJ: Erlbaum.

Edwards, L., \& Roper, T. J. (1982). Schedule-induced running in gerbils. Behaviour Analysis Letters, 2, 205212.

Falk, J. L. (1966). The motivational properties of schedule-induced polydipsia. Journal of the Experimental Analysis of Behavior, 9, 19-25.

Falk, J. L. (1971). The nature and determinants of adjunctive behavior. Physiology and Behavior, 6, 577588.

Freed, W. J., Zec, R. F., \& Mendelson, J. (1977). Schedule-induced polydipsia: The role of orolingual factors and a new hypothesis. In J. A. W. M. Weijnen \& J. Mendelson (Eds.), Drinking behavior: Oral stimulation, reinforcement, and preference (pp. 341-397). New York: Plenum Press.

Hinson, J. M., \& Staddon, J. E. R. (1978). Behavioral competition: A mechanism for schedule interaction. Science, 202, 432-434.

Killeen, P. (1975). On the temporal control of behavior. Psychological Review, 82, 89-115.

Killeen, P. (1979). Arousal: Its genesis, modulation, and extinction. In M. D. Zeiler \& P. Harzem (Eds.), Advances in analysis of behavior: Vol. 1. Reinforcement and the organization of behavior (pp. 31-78). Chichester, England: Wiley.

King, G. D. (1974). Wheel running in the rat induced by a fixed-time presentation of water. Animal Learning of Behavior, 2, 325-328.

Knutson, J. F., \& Schrader, S. P. (1975). A concurrent assessment of schedule-induced aggression and schedule-induced polydipsia in the rat. Animal Learning of Behavior, 3, 16-20.

Lucas, G. A., Timberlake, W., \& Gawley, D. J. (1988). Adjunctive behavior of the rat under periodic food delivery in a 24-hour environment. Animal Learning o Behavior, 16, 19-30.

McIntire, K., Lundervold, D., Calmes, H., Jones, C., \&
Allard, S. (1983). Temporal control in a complex environment: An analysis of schedule-related behavior. Journal of the Experimental Analysis of Behavior, 39, 465-478.

Osborne, S. R. (1978). A quantitative analysis of the effects of amount of reinforcement on two response classes. Journal of Experimental Psychology: Animal Behavior Processes, 4, 297-317.

Reberg, D., Mann, B., \& Innis, N. K. (1977). Superstitious behavior for food and water in the rat. Physiology and Behavior, 19, 803-806.

Reid, A. K., \& Dale, R. H. I. (1983). Dynamic effects of food magnitude on interim-terminal interaction. Journal of the Experimental Analysis of Behavior, 39, 135-148.

Reid, A. K., Piñones Vazquez, P., \& Alatorre Rico, J. (1985). Schedule induction and the temporal distributions of adjunctive behavior on periodic water schedules. Animal Learning \& Behavior, 13, 321-326.

Reid, A. K., \& Staddon, J. E. R. (1982). Scheduleinduced drinking: Elicitation, anticipation, or behavioral interaction? Journal of the Experimental Analysis of Behavior, 38, 1-18.

Reid, A. K., \& Staddon, J. E. R. (1990). Mechanisms of schedule entrainment. In S. J. Cooper \& C. T. Dourish (Eds.), Neurobiology of stereotyped behaviour (pp. 200-231). Oxford: Oxford University Press.

Riley, A. L., Peele, D. B., Richard, K. D., \& Kulkosky, P. J. (1981). The interaction of conditioned taste aversions and schedule-induced polydipsia: Availability of alternative behaviors. Animal Learning \& Behavior, 9, 287-290.

Roper, T. J. (1978). Diversity and substitutability of adjunctive activities under fixed-interval schedules of food reinforcement. Journal of the Experimental Analysis of Behavior, 30, 83-96.

Roper, T. J., Edwards, L., \& Crossland, G. (1983). Factors affecting schedule induced wood-chewing in rats: Percentage and the rate of reinforcement, and operant requirement. Animal Learning \& Behavior, 11, 35-43.

Rosellini, R. A. (1985). An application of opponentprocess theory to adjunctive behavior. In F. R. Brush \& J. B. Overmeier (Eds.), Affect, conditioning, and cognition: Essays on determinants of behavior (pp. 263-280). Hillsdale, NJ: Erlbaum.

Segal, E. F. (1969). The interaction of psychogenic polydipsia with wheel running in rats. Psychonomic Science, 14, 141-144.

Staddon, J. E. R. (1977). Schedule-induced behavior. In W. K. Honig \& J. E. R. Staddon (Eds.), Handbook of operant behavior (pp. 125-152). Englewood Cliffs, NJ: Prentice-Hall.

Staddon, J. E. R., \& Ayres, S. L. (1975). Sequential and temporal properties of behavior induced by a schedule of periodic food delivery. Behaviour, 54, 2649.

Thompson, T., \& Lubinski, D. (1986). Units of analysis and kinetic structure of behavioral repertoires. Journal of the Experimental Analysis of Behavior, 46, 219-242.

Timberlake, W. (1990). Natural learning in laboratory paradigms. In D. A. Dewsbury (Ed.), Contemporary issues in comparative psychology (pp. 31-54). Sunderland, MA: Sinauer.

Timberlake, W., \& Lucas, G. A. (1989). Behavior sys- 
tems and learning: From misbehavior to general laws. In S. B. Klein \& R. R. Mowrer (Eds.), Contemporary learning theories: Instrumental conditioning theory and the impact of biological constraints on learning (pp. 237275). Hillsdale, NJ: Erlbaum.

Wetherington, C. L., \& Riley, A. L. (1986). Schedule- induced polydipsia: Interactions with wheel running. Animal Learning \& Behavior, 14, 416-420.

Received July 30, 1991 Final acceptance July 31, 1992 